\title{
ERROR ANALYSIS OF FULLY DISCRETE VELOCITY-CORRECTION METHODS FOR INCOMPRESSIBLE FLOWS
}

\author{
J. L. GUERMOND, JIE SHEN, AND XIAOFENG YANG
}

\begin{abstract}
A fully discrete version of the velocity-correction method, proposed by Guermond and Shen (2003) for the time-dependent Navier-Stokes equations, is introduced and analyzed. It is shown that, when accounting for space discretization, additional consistency terms, which vanish when space is not discretized, have to be added to establish stability and optimal convergence. Error estimates are derived for both the standard version and the rotational version of the method. These error estimates are consistent with those by Guermond and Shen (2003) as far as time discretiztion is concerned and are optimal in space for finite elements satisfying the inf-sup condition.
\end{abstract}

\section{INTRODUCTION}

Projection methods, whose original version was introduced by Chorin [3] and Temam [27] in the late 1960s, are widely used to approximate the incompressible time-dependent Navier-Stokes equations. They are designed to overcome the difficulty caused by the incompressibility constraint which couples the velocity and the pressure. We refer to a recent review on this topic 13 where projection schemes are classified into three families: pressure-correction (cf. e.g. 4, 7, 14, 20, 23, 26, 28, 29]), velocity-correction (cf. [12, 18, 19, 22]), and consistent splitting scheme [11, 17, 25] (which is equivalent, in the space continuous case only, to the so-called gauge method [5, 21]).

Velocity-correction schemes (in semi-discretized form) were first introduced in a disguised form in [22, 18, and rigorously analyzed by Guermond and Shen in [12]. The main difference between the velocity-correction methods and the pressurecorrection or the consistent-splitting methods is that, in velocity-correction methods, the viscous term is made explicit in the first sub-step and corrected in the second sub-step, whereas in the other methods it is the pressure gradient which is made explicit first and corrected afterward. In addition to convergence proofs on various semi-discretized forms of the velocity-correction scheme, numerical tests on a second-order fully discretized version of the method are also reported in 12 . These tests, using spectral and finite element methods, show that the method is

Received by the editor December 29, 2006 and, in revised form, June 3, 2007.

2000 Mathematics Subject Classification. Primary 65M12, 35Q30, 35J05, 76D05.

Key words and phrases. Navier-Stokes equations, velocity-correction, projection methods, finite element methods, spectral methods, incompressibility, fraction step methods.

The work of the first author was supported in part by NSF DMS-0510650.

The work of the second and third authors was supported in part by NSF DMS-0509665 and DMS-0610646.

(C) 2008 American Mathematical Society Reverts to public domain 28 years from publication 1387 
stable and yield quasi-optimal results in time and space for the velocity and the pressure. However, to the best of our knowledge, there is no further work in the literature that provides a rigorous stability and error analysis for the fully discretized method, using either finite element or spectral approximation in space.

A rather general strategy for analyzing various two-step projection methods has been devised in $[8]$. The main ingredient of this theory is to consider two different approximation spaces for the velocity, one for each sub-step. Using the notations from [8, the velocity approximation in the viscous sub-step is chosen in a finitedimensional space $X_{h}$, and that in the projection sub-step is chosen in another finite-dimensional space $Y_{h}$ which contains $X_{h}$. For the special choice of $Y_{h}=X_{h}$, there is no essential difference in the analysis between the fully discrete case and the semi-discrete case; that is to say, all the arguments from [12 carry over to the fully discrete situation naturally. However, the situation $X_{h}=Y_{h}$ implies that the pressure is computed by solving a Darcy problem in mixed form. In order to compute the pressure by solving a Poisson problem, thus avoiding a possibly awkward Darcy problem, we have to look at situations where $X_{h} \neq Y_{h}$. However, if one naively uses the semi-discrete forms of the algorithm using $X_{h} \neq Y_{h}$, one observes a subtle inconsistency, especially for the rotational form of the scheme, which makes it very difficult, if not impossible, to prove the stability and optimal convergence of the fully discretized scheme. The primary goal of the present paper is to construct a fully discrete velocity-correction scheme which removes the inconsistency mentioned above. This is done by adding terms that vanish when the space is continuous and when $Y_{h}=X_{h}$. A particular instance of the fully discretized method that we propose consists of solving a discrete (standard) Poisson equation for the pressure.

The paper is organized as follows. In $₫ 2$ we introduce notation and the discrete setting for the space approximation. In 3 we discuss how the velocity-correction algorithm in standard form should be discretized in space and show in particular that naively discretizing the semi-discrete algorithm yields inconsistencies as mentioned above. In 4 we prove stability and convergence for the first-order rotational velocity-correction scheme. In $\$ 5$ we study the second-order version of the rotational velocity-correction scheme. The two major results of this paper are Theorems 4.1 and 5.1. Concluding remarks are reported in $\$ 6$.

\section{Preliminaries}

2.1. The continuous problem. Since it is well known that non-linear terms in the Navier-Stokes equations do not affect the formal accuracy of fractional-step projection methods provided they are consistently treated, we henceforth restrict ourselves to the time-dependent Stokes problem:

$$
\left\{\begin{array}{l}
\partial_{t} u-\nabla^{2} u+\nabla p=f \quad \text { in } \Omega \times[0, T], \\
\operatorname{div} u=0 \quad \text { in } \Omega \times[0, T]
\end{array}\right.
$$

supplemented with initial and, for simplicity, homogeneous Dirichlet boundary conditions

$$
\left.u\right|_{t=0}=v_{0} \quad \text { in } \Omega,\left.\quad u\right|_{\partial \Omega}=0 .
$$

In the above problem, $f \in L^{2}((0, T) \times \Omega)$ is a body force, and $\Omega$ is an open bounded domain in $R^{d}(d=2$ or 3 ) with a boundary sufficiently smooth so that the usual 
$H^{2}$ regularity holds for the steady Stokes problem with homogeneous Dirichlet boundary conditions and a source term in $L^{2}(\Omega)$. The symbol $\partial_{t}$ denotes the partial derivative with respect to time. We also use $d_{t}$ in the rest of the paper to denote derivatives with respect to time.

We denote by $W^{s, p}(\Omega)$ and $W_{0}^{s, p}(\Omega)$ the usual Sobolev spaces equipped with the norm $\|\cdot\|_{s, p}$ for $0 \leq s \leq \infty, 1 \leq p \leq \infty$. In particular, we denote the Hilbert spaces $W^{s, 2}(\Omega)$ by $H^{s}(\Omega)(s=0, \pm 1, \ldots)$ with norm $\|\cdot\|_{s}$ and semi norm $|\cdot|_{s}$. The norm and inner product of $L^{2}(\Omega)=H^{0}(\Omega)$ are denoted by $\|\cdot\|_{0}$ and $(\cdot, \cdot)$ respectively.

We shall also make use of the following Hilbert spaces:

$$
\begin{aligned}
& L_{f=0}^{2}(\Omega)=\left\{q \in L^{2}(\Omega), \int_{\Omega} q=0\right\}, \\
& H_{f=0}^{1}(\Omega)=\left\{q \in H^{1}(\Omega), \int_{\Omega} q=0\right\}, \\
& H=\left\{v \in L^{2}(\Omega)^{d}, \nabla \cdot v=0,\left.v \cdot n\right|_{\Gamma}=0\right\} .
\end{aligned}
$$

In particular, the following Helmholtz decomposition of $L^{2}(\Omega)^{d}$ plays an important role for the analysis of projection methods:

$$
L^{2}(\Omega)^{d}=H \oplus \nabla H_{f=0}^{1}(\Omega) .
$$

2.2. The discrete setting. Let $\delta t>0$ be a real number that we henceforth refer to as the time step. We set $t^{k}=k \delta t$ for $0 \leq k \leq K=[T / \delta t]$. For every function which is continuous in time, $\phi(t)$, we denote $\phi^{k}:=\phi\left(t^{k}\right)$ and define the difference operator $\delta$, acting on sequences, by $\delta \phi^{k}:=\phi^{k}-\phi^{k-1}$. Let $W$ be a Banach space; we set $L^{p}(W)=L^{p}(0, T ; W)$. To account for time sequences we also set $\ell^{p}(W):=\left\{w=\left(w^{0}, w^{1}, \ldots, w^{K}\right), w^{k} \in W, 0 \leq k \leq K,\|\phi\|_{\ell^{p}(W)}<+\infty\right\}$ with

$$
\|\phi\|_{\ell^{p}(W)}:=\left(\delta t \sum_{k=0}^{K}\left\|\phi^{k}\right\|_{W}^{p}\right)^{\frac{1}{p}}, \quad\|\phi\|_{\ell^{\infty}(W)}:=\max _{0 \leq k \leq K}\left(\left\|\phi^{k}\right\|_{W}\right) .
$$

Let $\left\{X_{h}\right\}_{h>0},\left\{M_{h}\right\}_{h>0}$ be two families of conforming approximations of $H_{0}^{1}(\Omega)^{d}$ and $L_{0}^{2}(\Omega)$, respectively. The pair $\left(X_{h}, M_{h}\right)$ is assumed to be compatible in the sense that the following LBB conditions hold uniformly with respect to $h$ :

$$
\exists c>0, \quad \inf _{q_{h} \in M_{h}} \sup _{v_{h} \in X_{h}} \frac{\left(\nabla \cdot v_{h}, q_{h}\right)}{\left\|\nabla v_{h}\right\|_{0}} \geq c\left\|q_{h}\right\|_{0} .
$$

We henceforth denote by $c$ a generic constant that is independent of the mesh-size $h$ and the time step $\delta t$ but possibly depends on the data and the solution. Whenever no confusion is possible we use the expression $A \lesssim B$ to say that there exists a generic constant $c$ such that $A \leq c B$.

The two (families of) spaces $X_{h}$ and $M_{h}$ are also assumed to satisfy the following approximation properties: There exists an integer $l>0$ such that for all $r \in[1, l]$,

$$
\begin{gathered}
\inf _{v_{h} \in X_{h}}\left\{\left\|v-v_{h}\right\|_{0}+h\left\|v-v_{h}\right\|_{1}\right\} \lesssim h^{r+1}\|v\|_{r+1}, \quad \forall v \in H^{r+1}(\Omega)^{d} \cap H_{0}^{1}(\Omega)^{d} . \\
\inf _{q_{h} \in Q_{h}}\left\{\left\|q-q_{h}\right\|_{0}+h\left\|q-q_{h}\right\|_{1}\right\} \lesssim h^{r}\|q\|_{r}, \quad \forall q \in H^{r}(\Omega) \cap L_{0}^{2}(\Omega) .
\end{gathered}
$$

In order to formulate the semi-discrete Stokes problem in a way which is similar to its continuous differential counterpart, we introduce several discrete differential operators as in 8 . We define the discrete Laplace operator, $A_{h}: X_{h} \rightarrow X_{h}^{\prime}$, by

$$
\left(A_{h} u_{h}, v_{h}\right)=\left(\nabla u_{h}, \nabla v_{h}\right), \quad \forall\left(u_{h}, v_{h}\right) \in X_{h} \times X_{h},
$$


the discrete divergence operator, $B_{h}: X_{h} \rightarrow M_{h}$, and the discrete gradient operator, $B_{h}^{T}: M_{h} \rightarrow X_{h}^{\prime}$, by

$$
\left(B_{h} v_{h}, p_{h}\right)=-\left(\nabla \cdot v_{h}, p_{h}\right)=\left(v_{h}, B_{h}^{T} p_{h}\right), \quad \forall\left(v_{h}, p_{h}\right) \in X_{h} \times M_{h} .
$$

We also define an extension of the $L^{2}$-projection onto $X_{h}, \pi_{h}: H^{-1}(\Omega)^{d} \rightarrow X_{h}^{\prime}$ such that

$$
\left(\pi_{h} f, v_{h}\right)=\left(f, v_{h}\right), \forall v_{h} \in X_{h} .
$$

Using the discrete framework defined above, the time-dependent Stokes problem (2.1) can be semi-discretized as follows: Setting $f_{h}=\pi_{h} f$ and $v_{0, h}=\pi_{h} u_{0}$, we look for $u_{h}(t) \in \mathcal{C}^{0}\left([0, T] ; X_{h}\right)$ and $p_{h}(t) \in L^{2}\left((0, T) ; M_{h}\right)$ such that

$$
\left\{\begin{array}{l}
\frac{d u_{h}}{d t}+A_{h} u_{h}+B_{h}^{T} p_{h}=f_{h}, \quad 0<t \leq T, \\
B_{h} u_{h}=0, \\
\left.u_{h}\right|_{t=0}=v_{0, h} .
\end{array}\right.
$$

It is well known that the above problem admits a unique solution which is stable with respect to the data. Furthermore, since $X_{h}$ and $M_{h}$ are convergent and stable approximations of $H_{0}^{1}(\Omega)^{d}$ and $H_{f=0}^{1}(\Omega)$, the solution to (2.14) converges in an appropriate sense to that of the continuous problem (2.1). For more details on the above formulation using finite elements we refer to [6, 15, 16].

2.3. The $\left(X_{h}, Y_{h}\right)$ pair. Following Guermond [8, we introduce an additional discrete setting so as to relax the incompressibility constraint and to build a discrete version of the Helmholtz decomposition (2.6). More precisely, we want to decompose each discrete vector field $\tilde{u}_{h} \in X_{h}$ into the sum of a discrete-divergencefree vector field $u_{h}$ plus the discrete-gradient of a scalar field $\phi_{h}$ in $M_{h}$. There are numerous ways of achieving this decomposition. For instance, we could set $\tilde{u}_{h}=u_{h}+B_{h}^{T} \phi_{h}$, with $u_{h} \in X_{h}$ and $B_{h} u_{h}=0$. Another possibility could be to set $\tilde{u}_{h}=u_{h}+\nabla \phi_{h}$ where $u_{h}$ is enforced to be orthogonal to $\nabla M_{h}$, provided $M_{h}$ is constructed so that $M_{h} \subset H_{f=0}^{1}(\Omega)$. In this case it is natural to choose $u_{h}$ to be in $X_{h}+\nabla M_{h}$. Even though this alternative may seem odd, it turns out to be optimal and very easy to implement, since it implies solving a discrete Poisson problem using the usual $\left(\nabla \phi_{h}, \nabla \psi_{h}\right)$ bilinear form.

In order to present a unified analysis for the many possible realizations of the discrete Helmholtz decomposition, we introduce a finite dimensional subspace $Y_{h} \in$ $L^{2}(\Omega)^{d}$. For the sake of simplicity we assume that $X_{h} \subset Y_{h}$ and we denote by $i_{h}$ the continuous injection of $X_{h}$ into $Y_{h}$; the transpose of $i_{h}$ is the $L^{2}$-projection of $Y_{h}$ onto $X_{h}$. Furthermore, we assume that we have at hand an operator $C_{h}: Y_{h} \longrightarrow M_{h}$ which is an extension of $B_{h}$, i.e.,

$$
C_{h} i_{h}=B_{h}, \quad i_{h}^{T} C_{h}^{T}=B_{h}^{T} .
$$

Owing to (2.8), $B_{h}$ is surjective. $C_{h}$ being an extension of $B_{h}$, this immediately implies that $C_{h}$ is also surjective and $C_{h}^{T}$ is injective. As a result $\left\|C_{h}^{T} q\right\|_{0}$ is a norm and, upon setting $H_{h}=\mathrm{KERC}_{h}$, the following orthogonal decomposition of $Y_{h}$ holds:

$$
Y_{h}=H_{h} \oplus C_{h}^{T}\left(M_{h}\right) .
$$


This decomposition is a discrete counterpart of (2.6). Finally, we also assume that $A_{h}$ and $C_{h}$ satisfy the following hypotheses:

$$
\begin{aligned}
\forall v_{h} \in X_{h}, \forall v \in\left[H_{0}^{1}(\Omega) \cap H^{2}(\Omega)\right]^{d}, & \left(\left\|v_{h}-v\right\|_{1} \lesssim h\|v\|_{2}\right) \Rightarrow\left\|A_{h} v_{h}\right\|_{0} \lesssim\|v\|_{2}, \\
\forall q_{h} \in M_{h}, \forall q \in H_{j=0}^{1}(\Omega), & \left(\left\|q_{h}-q\right\|_{0} \lesssim h\|q\|_{1}\right) \Rightarrow\left\|C_{h}^{T} q_{h}\right\|_{0} \lesssim\|q\|_{1} .
\end{aligned}
$$

These hypotheses are usually satisfied when $X_{h}, Y_{h}$, and $M_{h}$ are constructed using finite elements with shape-regular meshes.

Various realizations of $Y_{h}$ and $C_{h}$ are described in [8, 10. An obvious one is $Y_{h}=$ $X_{h}$ and $C_{h}=B_{h}$. Assuming $M_{h} \subset H_{f=0}^{1}(\Omega)$, another interesting choice consists of setting $Y_{h}=X_{h}+\nabla M_{h}$ and defining $C_{h}$ such that $\left(C_{h} v_{h}, q_{h}\right)=\left(v_{h}, \nabla q_{h}\right)=$ $\left(v_{h}, C_{h}^{T} q_{h}\right)$, for all $v_{h} \in Y_{h}, q_{h} \in M_{h}$. This particular setting implies that $C_{h}^{T}$ is the restriction of $\nabla$ to $M_{h}$, i.e., $C_{h}^{T} q_{h}=\nabla q_{h}, \forall q_{h} \in M_{h}$. In particular, the bilinear form $\left(C_{h}^{T} q_{h}, C_{h}^{T} r_{h}\right)$ reduces to the usual weak form $\left(\nabla q_{h}, \nabla r_{h}\right)$ associated with the Poisson problem supplemented with Neumann boundary conditions, which is really easy to implement.

\section{FUlLy DisCRETIZED VELOCITY-CORRECTION IN STANDARD FORM}

3.1. A naive discretization. Consider for the time being the first-order backward Euler method. The standard velocity-correction scheme proposed in 12 in semidiscrete form is as follows: Set $u^{0}=u\left(t^{0}\right)$, then for $k \geq 0$, compute $u^{k+1} \in H$ and $p^{k+1} \in L_{0}^{2}(\Omega)$ such that

$$
\left\{\begin{array}{l}
\frac{u^{k+1}-\tilde{u}^{k}}{\delta t}-\nabla^{2} \tilde{u}^{k}+\nabla p^{k+1}=f\left(t^{k+1}\right), \\
\nabla \cdot u^{k+1}=0,\left.\quad u^{k+1} \cdot n\right|_{\Gamma}=0
\end{array}\right.
$$

and then find $\tilde{u}^{k+1} \in H_{0}^{1}(\Omega)^{d}$ such that

$$
\frac{\tilde{u}^{k+1}-u^{k+1}}{\delta t}-\nabla^{2}\left(\tilde{u}^{k+1}-\tilde{u}^{k}\right)=0,\left.\quad \tilde{u}^{k+1}\right|_{\Gamma}=0 .
$$

A seemingly natural way to discretize the above algorithm in space is as follows: Setting $\tilde{u}_{h}^{0}=\pi_{h} u_{0}$ and $f_{h}^{k+1}=\pi_{h} f\left(t^{k+1}\right)$, for $k \geq 1$, compute $\left(u_{h}^{k+1}, p_{h}^{k+1}\right) \in$ $Y_{h} \times M_{h}$ such that

$$
\left\{\begin{array}{l}
\frac{u_{h}^{k+1}-i_{h} \tilde{u}_{h}^{k}}{\delta t}+i_{h} A_{h} \tilde{u}_{h}^{k}+C_{h}^{T} p_{h}^{k+1}=i_{h} f_{h}^{k+1}, \\
C_{h} u_{h}^{k+1}=0
\end{array}\right.
$$

and then compute $\tilde{u}_{h}^{k+1} \in X_{h}$ such that

$$
\frac{\tilde{u}_{h}^{k+1}-i_{h}^{T} u_{h}^{k+1}}{\delta t}+A_{h} \tilde{u}_{h}^{k+1}-A_{h} \tilde{u}_{h}^{k}=0 .
$$

Let us now assume that this algorithm converges to a steady state as $k \rightarrow \infty$. Then (3.4) yields $\tilde{u}_{h}=i_{h}^{T} u_{h}$, which in turn implies $B_{h} \tilde{u}_{h}=B_{h} i_{h}^{T} u_{h}$. Therefore, we usually have $B_{h} \tilde{u}_{h} \neq 0$ unless $B_{h} i_{h}^{T} u_{h}=C_{h} u_{h}$, which is true only if $i_{h}^{T}$ is the identity operator and $B_{h}=C_{h}$. Observe that the equality $B_{h}=C_{h}$ holds only if $X_{h}=Y_{h}$. We then conclude that (3.3)-(3.4) is consistent only if $X_{h}=Y_{h}$, which greatly reduces implementation options. As a result, one must find a consistent 
way to discretize (3.1)-(3.2) in order to use more convenient implementation options for which $X_{h} \neq Y_{h}$. This is one of the main goals of the present paper.

3.2. Consistent discretization. The above observation led us to consider the following alternative discretization of (3.1)-(3.2):

$$
\left\{\begin{array}{l}
\frac{u_{h}^{k+1}-i_{h} \tilde{u}_{h}^{k}}{\delta t}+i_{h} A_{h} \tilde{u}_{h}^{k}+C_{h}^{T} p_{h}^{k+1}+i_{h} B_{h}^{T} p_{h}^{k}-C_{h}^{T} p_{h}^{k}=i_{h} f_{h}^{k+1}, \\
C_{h} u_{h}^{k+1}=0
\end{array}\right.
$$

and

$$
\frac{\tilde{u}_{h}^{k+1}-i_{h}^{T} u_{h}^{k+1}}{\delta t}+A_{h} \tilde{u}_{h}^{k+1}-A_{h} \tilde{u}_{h}^{k}=0
$$

Clearly, the term $i_{h} B_{h}^{T} p_{h}^{k}-C_{h}^{T} p_{h}^{k}$ in (3.5) vanishes when $X_{h}=Y_{h}$, implying that the above algorithm is the same as (3.3)-(3.4) when $X_{h}=Y_{h}$. Let us observe also that (3.6) can be rewritten in another equivalent form as follows: Applying $i_{h}^{T}$ to (3.5) and adding the result to (3.6). Upon noticing that $i_{h}^{T} i_{h} B_{h}^{T} p_{h}^{k}=i_{h}^{T} C_{h}^{T} p_{h}^{k}$ thanks to (2.15) and the fact that $\left.i_{h}^{T} i_{h}\right|_{X_{h}}$ is the identity on $X_{h}$, we obtain

$$
\frac{\tilde{u}_{h}^{k+1}-\tilde{u}_{h}^{k}}{\delta t}+A_{h} \tilde{u}_{h}^{k+1}+B_{h}^{T} p_{h}^{k+1}=f_{h}^{k+1},
$$

which is equivalent to (3.6). To understand why the new algorithm (3.5)-(3.7) (or (3.5)-(3.6), equivalently) is better than (3.3)-(3.4) in general, let us apply $i_{h}$ to (3.7) at time step $t^{k}$ and subtract the result from (3.5), giving

$$
\frac{u_{h}^{k+1}-2 i_{h} \tilde{u}_{h}^{k}+i_{h} \tilde{u}_{h}^{k-1}}{\delta t}+C_{h}^{T}\left(p_{h}^{k+1}-p_{h}^{k}\right)=i_{h}\left(f_{h}^{k+1}-f_{h}^{k}\right) .
$$

Assuming that there is a steady state as $k \rightarrow \infty$, this equation implies $u_{h}=i_{h} \tilde{u}_{h}$, which in turn yields $0=C_{h} u_{h}=C_{h} i_{h} \tilde{u}_{h}=B_{h} \tilde{u}_{h}$, since $C_{h} i_{h}=B_{h}$ by definition of $C_{h}$ being an extension of $B_{h}$. In other words, at steady state we have the desired property $B_{h} \tilde{u}_{h}=0$, which suggests that (3.5)-(3.7) is a consistent way of implementing (3.1)-(3.2). Actually, the above manipulation yields an efficient way to implement (3.5)-(3.7) without computing $u_{h}^{k+1}$, which might live in an odd space (think of $Y_{h}=X_{h}+\nabla M_{h}$ for instance). Owing to the constraint $\left(u^{k+1}, C_{h}^{T} r_{h}\right)=0$ for all $r_{h} \in M_{h}$, (3.8) can be equivalently rewritten as

$$
\left(C_{h}^{T}\left(p_{h}^{k+1}-p_{h}^{k}\right), C_{h}^{T} r_{h}\right)=\left(f^{k+1}-f^{k}, B_{h}^{T} r_{h}\right)+\frac{1}{\delta t}\left(2 \tilde{u}_{h}^{k}-\tilde{u}_{h}^{k-1}, B_{h}^{T} r_{h}\right) .
$$

Hence the algorithm is simply composed of the two sub-steps (3.9)-(3.7). As a result, choosing $Y_{h}$ only amounts to selecting a realization of $C_{h}^{T}$ with which the user is comfortable. For instance, choosing $Y_{h}=X_{h}+\nabla M_{h}$ implies that (3.9) is a simple discrete Poisson problem using the standard bilinear form $(\nabla ., \nabla$.$) .$

Remark 3.1. Actually, the algorithm (3.9)-(3.7) is exactly what was proposed in 12 as an equivalent alternative to (3.1)-(3.2) in a semi-discrete setting (see (2.8)(2.9) in [12]). The Finite Element computations reported in [12] have been done using (3.9)-(3.7). When [12] was written, it was not clear that (2.8)-(2.9) from [12] and (3.1)-(3.2) could yield different fully discrete implementations. One goal of the present paper is to clarify this observation. 
Instead of using the Euler scheme, one can use a higher-order method. For instance, using the second-order backward difference formula (BDF2), the fully discrete velocity-correction scheme in standard form takes the following form:

$$
\left\{\begin{array}{l}
\frac{3 u_{h}^{k+1}-4 i_{h} \tilde{u}_{h}^{k}+i_{h} \tilde{u}_{h}^{k-1}}{\delta t}+i_{h} A_{h} \tilde{u}_{h}^{k}+C_{h}^{T} p_{h}^{k+1}+i_{h} B_{h}^{T} p_{h}^{k}-C_{h}^{T} p_{h}^{k}=i_{h} f_{h}^{k+1} \\
C_{h} u_{h}^{k+1}=0
\end{array}\right.
$$

and

$$
\frac{3 \tilde{u}_{h}^{k+1}-4 \tilde{u}_{h}^{k}+\tilde{u}_{h}^{k-1}}{\delta t}+A_{h} \tilde{u}_{h}^{k+1}+B_{h}^{T} p_{h}^{k+1}=f_{h}^{k+1} .
$$

We finish this section by stating the following convergence results.

Theorem 3.1. Let $u, p$ solve (2.1). Assume enough regularity is at hand for $u$ and $p$. Let $u_{h}, p_{h}$ solve (3.5) -(3.7), then

$$
\begin{array}{ll}
\left\|u-\tilde{u}_{h}\right\|_{\ell^{2}\left(L^{2}(\Omega)^{d}\right)} & +\left\|u-i_{h}^{T} u_{h}\right\|_{\ell^{2}\left(L^{2}(\Omega)^{d}\right)} \lesssim \delta t+h^{l+1}, \\
\left\|u-\tilde{u}_{h}\right\|_{l^{2}\left(H^{1}(\Omega)^{d}\right)} & +\left\|p-p_{h}\right\|_{l^{2}\left(L^{2}(\Omega)\right)} \lesssim \delta t+h^{l} .
\end{array}
$$

Let $u_{h}, p_{h}$ solve (3.10)-(3.11) and assume the scheme be appropriately initialized. Then

$$
\begin{aligned}
\left\|u-\tilde{u}_{h}\right\|_{\ell^{2}\left(L^{2}(\Omega)^{d}\right)} & +\left\|u-i_{h}^{T} u_{h}\right\|_{\ell^{2}\left(L^{2}(\Omega)^{d}\right)} \lesssim \delta t^{2}+h^{l+1}, \\
\left\|u-\tilde{u}_{h}\right\|_{\ell^{2}\left(H^{1}(\Omega)^{d}\right)} & +\left\|p-p_{h}\right\|_{\ell^{2}\left(L^{2}(\Omega)\right)} \lesssim \delta t+h^{l} .
\end{aligned}
$$

Proof. We omit the details since they are similar to those in the proof of the rotational version of the algorithm which is detailed in the next section.

Remark 3.2. The estimate (3.15) is one-order suboptimal with respect to $\delta t$. The suboptimality is sharp in the sense that it cannot be improved. The origin of this defect is an inconsistent/artificial boundary condition which is enforced by (3.2). This equation implies that at the boundary of the flow domain

$$
\left.\nabla^{2} \tilde{u}^{k+1} \cdot n\right|_{\Gamma}=\left.\nabla^{2} \tilde{u}^{k} \cdot n\right|_{\Gamma}=\ldots=\left.\nabla^{2} \tilde{u}^{0} \cdot n\right|_{\Gamma},
$$

which together with (3.1) in turns gives

$$
\left.\frac{\partial p}{\partial n}\right|_{\Gamma}=\left.\left(f\left(t^{k+1}\right)+\nabla^{2} \tilde{u}^{0}\right) \cdot n\right|_{\Gamma}
$$

It is obviously an artificial Neumann boundary condition on the pressure. This phenomenon is identical to what is observed for the standard form of the pressurecorrection scheme; see e.g. [10, 13, 26]. The accuracy of the scheme is limited to $\mathcal{O}(\delta t)$ by the numerical boundary layer induced by this inconsistent/artificial boundary condition. The $\mathcal{O}(\delta t)$ barrier can be (partially) overcome by considering the rotational form of the method which is discussed in the next section.

\section{FULLY DisCRETIZED VELOCITY-CORRECTION IN ROTATIONAL FORM}

In this section we focus our attention on the velocity-correction method in rotational form using the first-order Euler scheme. This allows us to concentrate on the main issues by bypassing the technical issues associated with higher-order schemes. This strategy is based on the observation made in 9] that the splitting error (i.e., the difference between the discrete solution and that from the equivalent one-step algorithm where the pressure is implicit and the discrete impressibility constraint 
is enforced) does not depend on the time stepping. The stability analysis of the BDF2 time stepping is done in the next section for completeness, but all the key ingredients of the method are detailed in the present section using the first-order Euler time stepping.

4.1. Consistent fully discretization. Consider the rotational velocity-correction scheme in differential form as introduced in [12]: Set $\tilde{u}^{0}=u\left(t^{0}\right)$ and for $k \geq 0$, find $u^{k+1} \in H, p^{k+1} \in L_{0}^{2}(\Omega)$, and $\tilde{u}^{k+1} \in H_{0}^{1}(\Omega)^{d}$ such that

$$
\left\{\begin{array}{l}
\frac{u^{k+1}-\tilde{u}^{k}}{\delta t}+\nabla \times \nabla \times \tilde{u}^{k}+\nabla p^{k+1}=f\left(t^{k+1}\right), \\
\nabla \cdot u^{k+1}=0,\left.\quad u^{k+1} \cdot n\right|_{\Gamma}=0
\end{array}\right.
$$

and

$$
\frac{\tilde{u}^{k+1}-u^{k+1}}{\delta t}-\nabla^{2} \tilde{u}^{k+1}-\nabla \times \nabla \times \tilde{u}^{k}=0,\left.\quad \tilde{u}^{k+1}\right|_{\Gamma}=0 .
$$

Since $\nabla \times \nabla \times \tilde{u}^{k}=-\nabla^{2} \tilde{u}^{k}+\nabla \nabla \cdot \tilde{u}^{k}$, a natural approximation of $\nabla \times \nabla \times \tilde{u}^{k}$ is $i_{h} A_{h} \tilde{u}_{h}^{k}-C_{h}^{T} B_{h} \tilde{u}_{h}^{k}$, leading to the following fully discretized scheme: Set $\tilde{u}_{h}^{0}=\pi_{h} u_{0}$, then compute $\tilde{u}_{h}^{k+1} \in X_{h}, p_{h}^{k+1} \in M_{h}$, and $u_{h}^{k+1} \in Y_{h}$ such that

$$
\left\{\begin{array}{l}
\frac{u_{h}^{k+1}-i_{h} \tilde{u}_{h}^{k}}{\delta t}+i_{h} A_{h} \tilde{u}_{h}^{k}-C_{h}^{T} B_{h} \tilde{u}_{h}^{k}+C_{h}^{T} p_{h}^{k+1}=i_{h} f_{h}^{k+1}, \\
C_{h} u_{h}^{k+1}=0
\end{array}\right.
$$

and

$$
\frac{\tilde{u}_{h}^{k+1}-i_{h}^{T} u_{h}^{k+1}}{\delta t}+A_{h} \tilde{u}_{h}^{k+1}-A_{h} \tilde{u}_{h}^{k}+B_{h}^{T} B_{h} \tilde{u}_{h}^{k}=0 .
$$

By proceeding as in $\$ 3.1$, one can show that this naive algorithm is not consistent at steady state.

Inspired by the discussion in $\$ 3.2$, we now consider the following modified algorithm: Compute $\tilde{u}_{h}^{k+1} \in X_{h}, p_{h}^{k+1} \in M_{h}$, and $u_{h}^{k+1} \in Y_{h}$ such that

$$
\left\{\begin{array}{l}
\frac{u_{h}^{k+1}-i_{h} \tilde{u}_{h}^{k}}{\delta t}+i_{h} A_{h} \tilde{u}_{h}^{k}-C_{h}^{T} B_{h} \tilde{u}_{h}^{k}+C_{h}^{T} p_{h}^{k+1}+i_{h} B_{h}^{T} p_{h}^{k}-C_{h}^{T} p_{h}^{k}=i_{h} f_{h}^{k+1} \\
C_{h} u_{h}^{k+1}=0
\end{array}\right.
$$

and

$$
\frac{\tilde{u}_{h}^{k+1}-i_{h}^{T} u_{h}^{k+1}}{\delta t}+A_{h} \tilde{u}_{h}^{k+1}-A_{h} \tilde{u}_{h}^{k}+B_{h}^{T} B_{h} \tilde{u}_{h}^{k}=0 .
$$

Again, by proceeding as in $\$ 3.2$ this algorithm can be rewritten in an entirely equivalent way so as to completely avoid computing the velocity $u_{h}^{k+1} \in Y_{h}$. To see this, let us apply $i_{h}^{T}$ to (4.5) and add the result to (4.6) to obtain

$$
\frac{\tilde{u}_{h}^{k+1}-\tilde{u}_{h}^{k}}{\delta t}+A_{h} \tilde{u}_{h}^{k+1}+B_{h}^{T} p_{h}^{k+1}=f_{h}^{k+1} .
$$

Note that we used the following properties: $\left.i_{h}^{T} i_{h}\right|_{X_{h}}$ is the identity and $C_{h} i_{h}=B_{h}$. Now applying $-i_{h}$ to (4.7) at time step $t^{k}$ and adding the result to (4.5) yields

$$
\frac{u_{h}^{k+1}-2 i_{h} \tilde{u}_{h}^{k}+i_{h} \tilde{u}_{h}^{k-1}}{\delta t}+C_{h}^{T}\left(p_{h}^{k+1}-p_{h}^{k}-B_{h} \tilde{u}_{h}^{k}\right)=i_{h}\left(f_{h}^{k+1}-f_{h}^{k-1}\right) .
$$


Then, owing to the constraint $C_{h} u_{h}^{k+1}=0$, this problem can be recast into the following form: Solve for $\phi_{h}^{k+1} \in M_{h}$ such that

$$
\left(C_{h}^{T} \phi_{h}^{k+1}, C_{h}^{T} r_{h}\right)=\left(f^{k+1}-f^{k}, B_{h}^{T} r_{h}\right)+\frac{1}{\delta t}\left(2 \tilde{u}_{h}^{k}-\tilde{u}_{h}^{k-1}, B_{h}^{T} r_{h}\right), \quad \forall r_{h} \in M_{h} .
$$

Then set

$$
p_{h}^{k+1}=\phi_{h}^{k+1}+p_{h}^{k}+B_{h} \tilde{u}_{h}^{k} .
$$

In conclusion, an efficient way of implementing this algorithm consists of solving (4.9)-(4.10)-(4.7) .

Remark 4.1. The algorithm (4.9)-(4.10)-(4.7) is the discrete counterpart of the algorithm (3.6)-(3.7)-(3.8) which was proposed in [12.

4.2. Error estimates for the first-order rotational scheme. Let us start by rewriting the algorithm in a way which is better suited for the error analysis. Inspired by the analysis in [12 where it is shown that one has to work with time increments to prove stability, we now construct the algorithm for the time increments of the discrete unknowns.

First, we define

$$
\phi_{h}^{k+1}:=\delta p_{h}^{k+1}-B_{h} \tilde{u}_{h}^{k}, \quad D_{h}^{t} \tilde{u}_{h}^{k+1}:=A_{h} \delta \tilde{u}_{h}^{k+1}+B_{h}^{T} B_{h} \tilde{u}_{h}^{k} .
$$

Then we apply the increment operator $\delta$ to (4.5), (4.6), and (4.7) to obtain

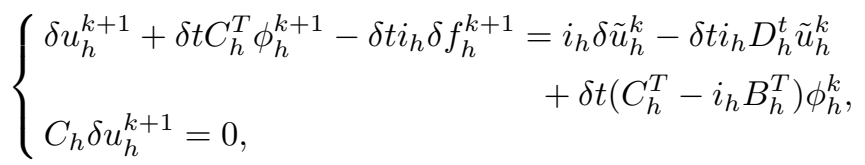

$$
\begin{aligned}
& \delta \tilde{u}_{h}^{k+1}+\delta t\left(D_{h}^{t} \tilde{u}_{h}^{k+1}-D_{h}^{t} \tilde{u}_{h}^{k}\right)=i_{h}^{T} \delta u_{h}^{k+1}, \\
& \delta \tilde{u}_{h}^{k+1}-\delta \tilde{u}_{h}^{k}+\delta t D_{h}^{t} \tilde{u}_{h}^{k+1}=-\delta t B_{h}^{T} \phi_{h}^{k+1}+\delta t \delta f_{h}^{k+1} .
\end{aligned}
$$

As usual we are going to compare $\left(u_{h}^{k}, p_{h}^{k}\right)$ with $\left(w_{h}\left(t^{k}\right), q_{h}\left(t^{k}\right)\right) \in X_{h} \times M_{h}$, which is the mixed approximation of $(u(t), p(t))$ defined as follows:

$$
\left\{\begin{array}{l}
\left(\nabla w_{h}(t), \nabla v_{h}\right)+\left(B_{h}^{T} q_{h}(t), v_{h}\right)=\left(\nabla u(t), \nabla v_{h}\right)-\left(p(t), \nabla \cdot v_{h}\right), \quad \forall v_{h} \in X_{h}, \\
\left(B_{h} w_{h}(t), r_{h}\right)=-\left(\nabla \cdot u(t), r_{h}\right), \quad \forall r_{h} \in M_{h} .
\end{array}\right.
$$

From the regularity properties of the Stokes problem, the following error estimates hold [9, 15].

Lemma 4.1. If $u^{(j)} \in L^{\beta}\left(H^{l+1}(\Omega)^{d}\right) \cap H_{0}^{1}(\Omega)^{d}, p^{(j)} \in L^{\beta}\left(H^{l}(\Omega)\right)$ for $1 \leq \beta \leq \infty$ and $j=0,1, \ldots$, then

$$
\left\|u^{(j)}-w_{h}^{(j)}\right\|_{L^{\beta}\left(L^{2}(\Omega)^{d}\right)}+h\left(\left\|u^{(j)}-w_{h}^{(j)}\right\|_{L^{\beta}\left(H^{1}(\Omega)^{d}\right)}+\left\|p^{(j)}-q_{h}^{(j)}\right\|_{L^{\beta}\left(L^{2}(\Omega)\right)}\right)
$$

We now rewrite (4.12)-(4.13)-(4.14) using $w_{h}$ and $q_{h}$. Owing to the definition of $\left(w_{h}(t), q_{h}(t)\right)$, the following identity holds at time $t=t^{k+1}$ :

$$
\left\{\begin{array}{l}
w_{h}^{k+1}+\delta t B_{h}^{T} q_{h}^{k+1}-\delta t f_{h}^{k+1}-\delta t R_{h}^{k+1}=w_{h}^{k}-\delta t A_{h} w_{h}^{k+1} \\
B_{h} w_{h}^{k+1}=0
\end{array}\right.
$$


where we have set $R_{h}^{k+1}=\frac{1}{\delta t}\left(w_{h}^{k+1}-w_{h}^{k}\right)-\pi_{h} \partial_{t} u^{k+1} \in X_{h}$. After applying $i_{h} \delta$ to (4.17), we obtain

$$
\left\{\begin{array}{l}
i_{h} \delta w_{h}^{k+1}+\delta t i_{h} B_{h}^{T} \delta q_{h}^{k+1}-\delta t i_{h} \delta f_{h}^{k+1}-i_{h} \delta R_{h}^{k+1}=i_{h} \delta w_{h}^{k}-\delta t i_{h} D_{h}^{t} w_{h}^{k+1}, \\
i_{h} B_{h} \delta w_{h}^{k+1}=0 .
\end{array}\right.
$$

Let us now introduce the following notation to denote various errors:

$$
\left\{\begin{array}{lll}
e_{h}^{k+1}=i_{h} w_{h}^{k+1}-u_{h}^{k+1}, & \tilde{e}_{h}^{k+1}=w_{h}^{k+1}-\tilde{u}_{h}^{k+1}, & \tilde{\psi}_{h}^{k+1}=\delta w_{h}^{k+1}+\tilde{e}_{h}^{k}, \\
\epsilon_{h}^{k+1}=q_{h}^{k+1}-p_{h}^{k+1}, & \varepsilon_{h}^{k+1}=\delta q_{h}^{k+1}-\phi_{h}^{k+1}, & \tilde{\phi}_{h}^{k+1}=\delta q_{h}^{k+1}-\phi_{h}^{k} .
\end{array}\right.
$$

Subtracting (4.12) from (4.18), we obtain

$$
\begin{cases}\delta e_{h}^{k+1}+\delta t C_{h}^{T} \varepsilon_{h}^{k+1}-\delta t i_{h} \delta R_{h}^{k+1}= & i_{h} \delta \tilde{e}_{h}^{k}-\delta t i_{h} D_{h}^{t} \tilde{\psi}_{h}^{k+1} \\ C_{h} \delta e_{h}^{k+1}=0 . & -\delta t i_{h} B_{h}^{T} \tilde{\phi}_{h}^{k+1}+\delta t C_{h}^{T} \tilde{\phi}_{h}^{k+1},\end{cases}
$$

After applying $\delta$ to (4.17) and subtracting (4.14) from it, we obtain

$$
\delta \tilde{e}_{h}^{k+1}-\delta \tilde{e}_{h}^{k}+\delta t D_{h}^{t} \tilde{e}_{h}^{k+1}=-\delta t B_{h}^{T} \varepsilon_{h}^{k+1}+\delta t \delta R_{h}^{k+1} .
$$

Now adding some zero terms to (4.13), we can rewrite it as

$$
\delta \tilde{e}_{h}^{k+1}+\delta t D_{h}^{t}\left(\tilde{e}_{h}^{k+1}-\tilde{\psi}_{h}^{k+1}\right)=i_{h}^{T} \delta e_{h}^{k+1} .
$$

The error analysis will be based entirely on the three equations (4.20)-(4.21)-(4.22).

Let us assume that the algorithm is initialized so that the following holds:

$$
\left\{\begin{array}{l}
\left\|\tilde{e}_{h}^{0}\right\|_{0} \lesssim \min \left(h^{l+1}, \delta t^{2} h^{l-1}\right), \quad\left\|\tilde{e}_{h}^{0}\right\|_{1} \lesssim \min \left(h^{l}, \delta t h^{l-1}\right), \\
\left\|A_{h} \tilde{e}_{h}^{0}\right\|_{0} \lesssim \min \left(h^{l-1}, \delta t h^{l-2}\right), \quad\left\|B_{h}^{t} B_{h} \tilde{e}_{h}^{0}\right\|_{0} \lesssim \min \left(h^{l-1}, \delta t h^{l-2}\right), \\
\left\|\epsilon_{h}^{0}\right\|_{1} \lesssim \min \left(h^{l-1}, \delta t h^{l-2}\right),
\end{array}\right.
$$

and the solution to (2.1) satisfies the following regularity hypothesis

$$
\begin{aligned}
& u, u_{t}, u_{t t} \in L^{2}\left(H^{l+1}(\Omega)^{d} \cap H_{0}^{1}(\Omega)^{d}\right), \quad u_{t t t} \in L^{2}\left(L^{2}(\Omega)^{d}\right) \\
& p \in L^{2}\left(H^{l}(\Omega)\right), \quad p_{t}, p_{t t} \in L^{2}\left(H^{1}(\Omega)\right) .
\end{aligned}
$$

Remark 4.2. If we set $\tilde{u}_{h}^{0}=w_{h}^{0}$ and $p_{h}^{0}=q_{h}^{0}$, then the hypothesis (H1) is naturally satisfied.

We are now in position to establish the first error estimate.

Lemma 4.2. If the hypotheses ( $(\mathrm{H1})-(\mathrm{H} 2)$ hold, we have

$$
\begin{aligned}
\left\|\delta \tilde{e}_{h}\right\|_{l^{\infty}\left(L^{2}(\Omega)^{d}\right)}+\left\|\tilde{e}_{h}-i_{h}^{T} e_{h}\right\|_{l^{\infty}\left(L^{2}(\Omega)^{d}\right)} & \lesssim \delta t\left(\delta t+h^{l+1}\right), \\
\left\|i_{h}^{T} \delta e_{h}-\delta \tilde{e}_{h}\right\|_{l^{2}\left(L^{2}(\Omega)^{d}\right)}+\left\|\delta^{2} \tilde{e}_{h}\right\|_{l^{2}\left(L^{2}(\Omega)^{d}\right)} & \lesssim \delta t^{5 / 2}+\delta t^{3 / 2} h^{l+1}, \\
\left\|B_{h} \tilde{u}_{h}\right\|_{l^{\infty}\left(L^{2}(\Omega)\right)} & \lesssim \delta t^{1 / 2}\left(\delta t+h^{l+1}\right) .
\end{aligned}
$$

Proof. Let us first recall a series of standard identities that will be used throughout the paper:

$$
\left\{\begin{array}{l}
2(a, b)=|a|^{2}+|b|^{2}-|a-b|^{2} \\
2(a-b, a)=|a|^{2}+|b|^{2}-|a-b|^{2}, \\
2(a-2 b+c, b)=\left(|a|^{2}-|b|^{2}\right)-\left(|b|^{2}-|c|^{2}\right)-|a-b|^{2}-|b-c|^{2} .
\end{array}\right.
$$


First, we square (4.20) and, noticing that $i_{h}^{T} C_{h}^{T} q=B_{h}^{T} q, \forall q \in M_{h}$ and $\left\|i_{h} v\right\|_{0}=$ $\|v\|_{0}, \forall v \in X_{h}$, we obtain

$$
\begin{aligned}
\left\|\delta e_{h}^{k+1}\right\|_{0}^{2} & +\delta t^{2}\left\|C_{h}^{T} \varepsilon_{h}^{k+1}\right\|_{0}^{2}+\delta t^{2}\left\|\delta R_{h}^{k+1}\right\|_{0}^{2} \\
& -2 \delta t\left(\delta e_{h}^{k+1}+\delta t C_{h}^{T} \varepsilon_{h}^{k+1}, i_{h} \delta R_{h}^{k+1}\right)=\left\|\delta \tilde{e}_{h}^{k}\right\|_{0}^{2}+\delta t^{2}\left\|D_{h}^{t} \tilde{\psi}_{h}^{k+1}\right\|_{0}^{2} \\
& -2 \delta t\left(i_{h} \delta \tilde{e}_{h}^{k}, i_{h} D_{h}^{t} \tilde{\psi}_{h}^{k+1}\right)+\delta t^{2}\left\|C_{h}^{T} \tilde{\phi}_{h}^{k+1}\right\|_{0}^{2}-\delta t^{2}\left\|B_{h}^{T} \tilde{\phi}_{h}^{k+1}\right\|_{0}^{2} .
\end{aligned}
$$

By the identities (4.26), we have

$$
\begin{aligned}
2 \delta t\left(i_{h} \delta \tilde{e}_{h}^{k}, i_{h} D_{h}^{t} \tilde{\psi}_{h}^{k+1}\right)= & 2 \delta t\left(\delta \tilde{e}_{h}^{k}, A_{h} \delta \tilde{\psi}_{h}^{k+1}+B_{h}^{T} B_{h} \tilde{\psi}_{h}^{k}\right) \\
= & 2 \delta t\left(\delta \tilde{e}_{h}^{k}, A_{h} \delta^{2} w_{h}^{k+1}+A_{h} \delta \tilde{e}_{h}^{k}+B_{h}^{T} B_{h} \tilde{e}_{h}^{k-1}\right) \\
= & \delta t\left(2\left\|\nabla \delta \tilde{e}_{h}^{k}\right\|_{0}^{2}-\left\|B_{h} \delta \tilde{e}_{h}^{k}\right\|_{0}^{2}\right)+\delta t\left(\left\|B_{h} \tilde{e}_{h}^{k}\right\|_{0}^{2}-\left\|B_{h} \tilde{e}_{h}^{k-1}\right\|_{0}^{2}\right) \\
& +2 \delta t\left(\delta \tilde{e}_{h}^{k}, A_{h} \delta^{2} w_{h}^{k+1}\right) .
\end{aligned}
$$

By substituting the above equality into (4.27), we infer

$$
\begin{aligned}
\left\|\delta e_{h}^{k+1}\right\|_{0}^{2} & +\delta t^{2}\left\|C_{h}^{T} \varepsilon_{h}^{k+1}\right\|_{0}^{2}+\delta t\left(2\left\|\nabla \delta \tilde{e}_{h}^{k}\right\|_{0}^{2}-\left\|B_{h} \delta \tilde{e}_{h}^{k}\right\|_{0}^{2}\right) \\
& +\delta t\left(\left\|B_{h} \tilde{e}_{h}^{k}\right\|_{0}^{2}-\left\|B_{h} \tilde{e}_{h}^{k-1}\right\|_{0}^{2}\right)=\left\|\delta \tilde{e}_{h}^{k}\right\|_{0}^{2}+\delta t^{2}\left\|D_{h}^{t} \tilde{\psi}_{h}^{k+1}\right\|_{0}^{2} \\
& +\delta t^{2}\left\|C_{h}^{T} \tilde{\phi}_{h}^{k+1}\right\|_{0}^{2}-\delta t^{2}\left\|B_{h}^{T} \tilde{\phi}_{h}^{k+1}\right\|_{0}^{2}+2 \delta t\left(i_{h}^{T} \delta e_{h}^{k+1}, \delta R_{h}^{k+1}\right) \\
& +2 \delta t^{2}\left(B_{h}^{T} \varepsilon_{h}^{k+1}, \delta R_{h}^{k+1}\right)-\delta t^{2}\left\|\delta R_{h}^{k+1}\right\|_{0}^{2}-2 \delta t\left(\delta \tilde{e}_{h}^{k}, A_{h} \delta^{2} w_{h}^{k+1}\right) .
\end{aligned}
$$

Next, we square (4.21) and use (4.26) to obtain

$$
\begin{aligned}
\left\|\delta^{2} \tilde{e}_{h}^{k+1}\right\|_{0}^{2} & +\delta t^{2}\left\|D_{h}^{t} \tilde{e}_{h}^{k+1}\right\|_{0}^{2}+\delta t\left(\left\|\nabla \delta \tilde{e}_{h}^{k+1}\right\|_{0}^{2}-\left\|\nabla \delta \tilde{e}_{h}^{k}\right\|_{0}^{2}\right) \\
& +\delta t\left\|\nabla \delta^{2} \tilde{e}_{h}^{k+1}\right\|_{0}^{2}+\delta t\left(\left\|B_{h} \tilde{e}_{h}^{k+1}\right\|_{0}^{2}-\left\|B_{h} \tilde{e}_{h}^{k}\right\|_{0}^{2}\right) \\
& -\delta t\left(\left\|B_{h} \tilde{e}_{h}^{k}\right\|_{0}^{2}-\left\|B_{h} \tilde{e}_{h}^{k-1}\right\|_{0}^{2}\right)-\delta t\left(\left\|B_{h} \delta \tilde{e}_{h}^{k+1}\right\|_{0}^{2}+\left\|B_{h} \delta \tilde{e}_{h}^{k}\right\|_{0}^{2}\right) \\
& =\delta t^{2}\left\|B_{h}^{T} \varepsilon_{h}^{k+1}\right\|_{0}^{2}+\delta t^{2}\left\|\delta R_{h}^{k+1}\right\|_{0}^{2}-2 \delta t^{2}\left(B_{h}^{T} \varepsilon_{h}^{k+1}, \delta R_{h}^{k+1}\right) .
\end{aligned}
$$

Then, we square (4.22) to obtain

$$
\begin{aligned}
\left\|\delta \tilde{e}_{h}^{k+1}\right\|_{0}^{2}+\delta t^{2} \| D_{h}^{t} \tilde{e}_{h}^{k+1} & -D_{h}^{t} \tilde{\psi}_{h}^{k+1} \|_{0}^{2} \\
& +2 \delta t\left(\delta \tilde{e}_{h}^{k+1}, D_{h}^{t} \tilde{e}_{h}^{k+1}-D_{h}^{t} \tilde{\psi}_{h}^{k+1}\right)=\left\|i_{h}^{T} \delta \tilde{e}_{h}^{k+1}\right\|_{0}^{2} .
\end{aligned}
$$

Notice that

$$
\begin{aligned}
2 \delta t\left(\delta \tilde{e}_{h}^{k+1}, D_{h}^{t} \tilde{e}_{h}^{k+1}-\right. & \left.D_{h}^{t} \tilde{\psi}_{h}^{k+1}\right)=2 \delta t\left(\delta \tilde{e}_{h}^{k+1}, D_{h}^{t} \delta \tilde{e}_{h}^{k+1}-D_{h}^{t} \delta w_{h}^{k+1}\right) \\
= & 2 \delta t\left(\nabla \delta \tilde{e}_{h}^{k+1}, \nabla \delta^{2} \tilde{e}_{h}^{k+1}\right)+2 \delta t\left(B{ }_{h} \delta \tilde{e}_{h}^{k+1}, B_{h} \delta \tilde{e}_{h}^{k}\right) \\
& -2 \delta t\left(\delta \tilde{e}_{h}^{k+1}, D_{h}^{t} \delta w_{h}^{k+1}\right) \\
= & \delta t\left(\left\|\nabla \delta \tilde{e}_{h}^{k+1}\right\|_{0}^{2}-\left\|\nabla \delta \tilde{e}_{h}^{k}\right\|_{0}^{2}+\left\|\nabla \delta^{2} \tilde{e}_{h}^{k+1}\right\|_{0}^{2}\right) \\
& +\delta t\left(\left\|B_{h} \delta \tilde{e}_{h}^{k+1}\right\|_{0}^{2}+\left\|B_{h} \delta \tilde{e}_{h}^{k}\right\|_{0}^{2}-\left\|B_{h} \delta^{2} \tilde{e}_{h}^{k+1}\right\|_{0}^{2}\right) \\
& -2 \delta t\left(\delta \tilde{e}_{h}^{k+1}, A_{h} \delta^{2} w_{h}^{k+1}\right),
\end{aligned}
$$

and

$$
\delta t^{2}\left\|D_{h}^{t} \tilde{e}_{h}^{k+1}-D_{h}^{t} \tilde{\psi}_{h}^{k+1}\right\|_{0}^{2}=\left\|i_{h}^{T} \delta e_{h}^{k+1}-\delta \tilde{e}_{h}^{k+1}\right\|_{0}^{2},
$$


so we can rewrite 4.30 as

$$
\begin{aligned}
\left\|\delta \tilde{e}_{h}^{k+1}\right\|_{0}^{2} & +\left\|i_{h}^{T} \delta e_{h}^{k+1}-\delta \tilde{e}_{h}^{k+1}\right\|_{0}^{2}+\delta t\left(\left\|\nabla \delta \tilde{e}_{h}^{k+1}\right\|_{0}^{2}-\left\|\nabla \delta \tilde{e}_{h}^{k}\right\|_{0}^{2}\right) \\
& +\delta t\left(\left\|B_{h} \delta \tilde{e}_{h}^{k+1}\right\|_{0}^{2}+\left\|B_{h} \delta \tilde{e}_{h}^{k}\right\|_{0}^{2}\right)+\delta t\left(\left\|\nabla \delta^{2} \tilde{e}_{h}^{k+1}\right\|_{0}^{2}-\left\|B_{h} \delta^{2} \tilde{e}_{h}^{k+1}\right\|_{0}^{2}\right) \\
& =\left\|i_{h}^{T} \delta e_{h}^{k+1}\right\|_{0}^{2}+2 \delta t\left(\delta \tilde{e}_{h}^{k+1}, A_{h} \delta^{2} w_{h}^{k+1}\right) .
\end{aligned}
$$

Now we add (4.28), (4.29), and (4.31). Note that the definition of $B_{h}$ together with the homogeneous Dirichlet boundary conditions on $v_{h}$ imply

$$
\left\|B_{h} v_{h}\right\|_{0}^{2} \leq\left\|\nabla \cdot v_{h}\right\|_{0}^{2} \leq\left\|\nabla \cdot v_{h}\right\|_{0}^{2}+\left\|\nabla \times v_{h}\right\|_{0}^{2}=\left\|\nabla v_{h}\right\|_{0}^{2}, \quad \forall v_{h} \in X_{h} .
$$

Observe, moreover, that $\left\|i_{h}^{T} u\right\|_{0} \leq\|u\|_{0}, \forall u \in Y_{h}$. These two facts then yield

$$
\begin{aligned}
\left\|\delta \tilde{e}_{h}^{k+1}\right\|_{0}^{2}+ & \delta t^{2}\left(\left\|C_{h}^{T} \varepsilon_{h}^{k+1}\right\|_{0}^{2}-\left\|B_{h}^{T} \varepsilon_{h}^{k+1}\right\|_{0}^{2}\right)+\delta t\left(\left\|B_{h} \tilde{e}_{h}^{k+1}\right\|_{0}^{2}-\left\|B_{h} \tilde{e}_{h}^{k}\right\|_{0}^{2}\right) \\
+ & \delta t^{2}\left\|D_{h}^{t} \tilde{e}_{h}^{k+1}\right\|_{0}^{2}+2 \delta t\left(\left\|\nabla \delta \tilde{e}_{h}^{k+1}\right\|_{0}^{2}-\left\|\nabla \delta \tilde{e}_{h}^{k}\right\|_{0}^{2}\right)+\left\|\delta^{2} \tilde{e}_{h}^{k+1}\right\|_{0}^{2} \\
+ & \left\|i_{h}^{T} \delta e_{h}^{k+1}-\delta \tilde{e}_{h}^{k+1}\right\|_{0}^{2}+\delta t\left\|\nabla \delta \tilde{e}_{h}^{k}\right\|_{0}^{2}+\delta t\left\|\nabla \delta^{2} \tilde{e}_{h}^{k+1}\right\|_{0}^{2} \\
\leq & \left\|\delta \tilde{e}_{h}^{k}\right\|_{0}^{2}+\delta t^{2}\left(\left\|C_{h}^{T} \tilde{\phi}_{h}^{k+1}\right\|_{0}^{2}-\left\|B_{h}^{T} \tilde{\phi}_{h}^{k+1}\right\|_{0}^{2}\right)+\delta t^{2}\left\|D_{h}^{t} \tilde{\psi}_{h}^{k+1}\right\|_{0}^{2} \\
& +2 \delta t\left(\delta^{2} \tilde{e}_{h}^{k+1}, A_{h} \delta^{2} w_{h}^{k+1}\right)+2 \delta t\left(\delta R_{h}^{k+1}, i_{h}^{T} \delta e_{h}^{k+1}\right) .
\end{aligned}
$$

We now derive bounds for the last four terms in the right-hand side.

The definition of $i_{h}^{T}$ implies

$$
\left\|i_{h} i_{h}^{T} v_{h}\right\|_{0}^{2}+\left\|v_{h}-i_{h} i_{h}^{T} v_{h}\right\|_{0}^{2}=\left\|v_{h}\right\|_{0}^{2}, \quad \forall v_{h} \in Y_{h} .
$$

Hence, from (2.15), we infer

$$
\begin{aligned}
\left\|C_{h}^{T} q_{h}\right\|_{0}^{2}-\left\|B_{h}^{T} q_{h}\right\|_{0}^{2} & =\left\|C_{h}^{T} q_{h}\right\|_{0}^{2}-\left\|i_{h} i_{h}^{T} C_{h}^{T} q_{h}\right\|_{0}^{2}=\left\|C_{h}^{T} q_{h}-i_{h} i_{h}^{T} C_{h}^{T} q_{h}\right\|_{0}^{2} \\
& =\left\|\left(C_{h}^{T}-i_{h} B_{h}^{T}\right) q_{h}\right\|_{0}^{2}, \quad \forall q_{h} \in M_{h} .
\end{aligned}
$$

Owing to this result together with the definition of $\tilde{\phi}_{h}$, we deduce that

$$
\begin{aligned}
\left\|C_{h}^{T} \tilde{\phi}_{h}^{k+1}\right\|_{0}^{2}-\left\|B_{h}^{T} \tilde{\phi}_{h}^{k+1}\right\|_{0}^{2} & =\left\|\left(C_{h}^{T}-i_{h} B_{h}^{T}\right)\left(\delta^{2} q_{h}^{k+1}+\varepsilon_{h}^{k}\right)\right\|_{0}^{2} \\
& =\left\|\left(C_{h}-i_{h} B_{h}^{T}\right) \delta^{2} q_{h}^{k+1}\right\|_{0}^{2}+\left\|\left(C_{h}-i_{h} B_{h}^{T}\right) \varepsilon_{h}^{k}\right\|_{0}^{2} \\
& +2\left(\left(C_{h}^{T}-i_{h} B_{h}^{T}\right) \delta^{2} q_{h}^{k+1},\left(C_{h}^{T}-i_{h} B_{h}^{T}\right) \varepsilon_{h}^{k}\right) .
\end{aligned}
$$

Then, using (2.18) together with Lemma 4.1, we obtain

$$
\left\|C_{h}^{T} \tilde{\phi}_{h}^{k+1}\right\|_{0}^{2}-\left\|B_{h}^{T} \tilde{\phi}_{h}^{k+1}\right\|_{0}^{2} \lesssim \delta t^{3}+(1+\delta t)\left\|\left(C_{h}-i_{h} B_{h}^{T}\right) \varepsilon_{h}^{k}\right\|_{0}^{2} .
$$

A bound on $\left\|D_{h}^{t} \tilde{\psi}_{h}^{k+1}\right\|_{0}^{2}$ can be obtained as follows:

$$
\left\|D_{h}^{t} \tilde{\psi}_{h}^{k+1}\right\|_{0}^{2} \leq\left(\left\|D_{h}^{t} \delta w_{h}^{k+1}\right\|_{0}+\left\|D_{h}^{t} \tilde{e}_{h}^{k}\right\|_{0}\right)^{2}=\left(\left\|A_{h} \delta^{2} w_{h}^{k+1}\right\|_{0}+\left\|D_{h}^{t} \tilde{e}_{h}^{k}\right\|_{0}\right)^{2} .
$$

Then using (2.17) together with Lemma 4.1, we obtain

$$
\left\|D_{h}^{t} \tilde{\psi}_{h}^{k+1}\right\|_{0}^{2} \lesssim \delta t^{3}+(1+\delta t)\left\|D_{h}^{t} \tilde{e}_{h}^{k}\right\|_{0}^{2} .
$$

For the two other terms from the right-hand side, upper bounds can be derived as follows:

$$
\begin{aligned}
2 \delta t\left(\delta R_{h}^{k+1}, i_{h}^{T} \delta e_{h}^{k+1}\right) & =2 \delta t\left(\delta R_{h}^{k+1}, i_{h}^{T} \delta e_{h}^{k+1}-\delta \tilde{e}_{h}^{k+1}\right)+2 \delta t\left(\delta R_{h}^{k+1}, \delta \tilde{e}_{h}^{k+1}\right) \\
& \leq 2 \delta t\left\|\delta R_{h}^{k+1}\right\|_{0}^{2}+\delta t\left\|i_{h}^{T} \delta e_{h}^{k+1}-\delta \tilde{e}_{h}^{k+1}\right\|_{0}^{2}+\delta t\left\|\delta \tilde{e}_{h}^{k+1}\right\|_{0}^{2} \\
& \lesssim \delta t\left(\delta t^{2}+\delta t h^{l+1}\right)^{2}+\delta t\left\|i_{h}^{T} \delta e_{h}^{k+1}-\delta \tilde{e}_{h}^{k+1}\right\|_{0}^{2}+\delta t\left\|\delta \tilde{e}_{h}^{k+1}\right\|_{0}^{2},
\end{aligned}
$$




$$
\begin{aligned}
2 \delta t\left(\delta^{2} \tilde{e}_{h}^{k+1}, D_{h}^{t} \delta w_{h}^{k+1}\right) & \leq \delta t\left\|\nabla \delta^{2} \tilde{e}_{h}^{k+1}\right\|_{0}^{2}+\delta t\left\|\nabla \delta^{2} w_{h}^{k+1}\right\|_{0}^{2} \\
& \lesssim \delta t\left\|\nabla \delta^{2} \tilde{e}_{h}^{k+1}\right\|_{0}^{2}+\delta t^{5} .
\end{aligned}
$$

Substituting all the inequalities above into (4.32), we obtain

$$
\begin{aligned}
(1-\delta t)\left\|\delta \tilde{e}_{h}^{k+1}\right\|_{0}^{2}+ & \delta t^{2}\left\|\left(C_{h}-i_{h} B_{h}^{T}\right) \varepsilon_{h}^{k+1}\right\|_{0}^{2}+\delta t\left\|B_{h} \tilde{e}_{h}^{k+1}\right\|_{0}^{2} \\
& +\delta t^{2}\left\|D_{h}^{t} \tilde{e}_{h}^{k+1}\right\|_{0}^{2}+2 \delta t\left\|\nabla \delta \tilde{e}_{h}^{k+1}\right\|_{0}^{2} \\
& +\left\|\delta^{2} \tilde{e}_{h}^{k+1}\right\|_{0}^{2}+(1-\delta t)\left\|i_{h}^{T} \delta e_{h}^{k+1}-\delta \tilde{e}_{h}^{k+1}\right\|_{0}^{2} \\
\lesssim & \left\|\delta \tilde{e}_{h}^{k}\right\|_{0}^{2}+\delta t^{2}(1+\delta t)\left\|\left(C_{h}^{T}-i_{h} B_{h}^{T}\right) \varepsilon_{h}^{k}\right\|_{0}^{2}+\delta t\left\|B_{h} \tilde{e}_{h}^{k}\right\|_{0}^{2} \\
& +(1+\delta t) \delta t^{2}\left\|D_{h}^{t} \tilde{e}_{h}^{k}\right\|_{0}^{2}+\delta t\left\|\nabla \delta \tilde{e}_{h}^{k}\right\|_{0}^{2}+\delta t^{3}\left(\delta t+h^{l+1}\right)^{2} .
\end{aligned}
$$

The discrete Gronwall lemma yields, for all $n \leq[T / \delta t]-1$,

$$
\begin{aligned}
\left\|\delta \tilde{e}_{h}^{n+1}\right\|_{0}^{2}+ & \delta t^{2}\left\|\left(C_{h}^{T}-i_{h} B_{h}^{T}\right) \varepsilon_{h}^{n+1}\right\|_{0}^{2}+\delta t\left\|B_{h} \tilde{e}_{h}^{n+1}\right\|_{0}^{2}+\delta t^{2}\left\|D_{h}^{t} \tilde{e}_{h}^{n+1}\right\|_{0}^{2} \\
& +\delta t\left\|\nabla \delta \tilde{e}_{h}^{k+1}\right\|_{0}^{2}+\sum_{k=1}^{n}\left(\left\|i_{h}^{T} \delta e_{h}^{k+1}-\delta \tilde{e}_{h}^{k+1}\right\|_{0}^{2}+\left\|\delta^{2} \tilde{e}_{h}^{k+1}\right\|_{0}^{2}\right) \\
\lesssim & \left\|\delta \tilde{e}_{h}^{1}\right\|_{0}^{2}+\delta t^{2}\left\|\left(C_{h}^{T}-i_{h} B_{h}^{T}\right) \varepsilon_{h}^{1}\right\|_{0}^{2}+\delta t\left\|B_{h} \tilde{e}_{h}^{1}\right\|_{0}^{2} \\
& +\delta t^{2}\left\|D_{h}^{t} \tilde{e}_{h}^{1}\right\|_{0}^{2}+\delta t\left\|\nabla \delta \tilde{e}_{h}^{1}\right\|_{0}^{2}+\delta t^{2}\left(\delta t+h^{l+1}\right)^{2} .
\end{aligned}
$$

In order to estimate the initial error terms in the right-hand side of the above inequality we make use of (4.17)-(4.6) at the first time step (i.e., $k=0$ ). More precisely, the equations that control $e_{h}^{1}, \tilde{e}_{h}^{1}$, and $\varepsilon_{h}^{1}$ are obtained by subtracting (4.5) from the equation obtained by applying $i_{h}$ to (4.17), and adding some zero terms to (4.6) as follows:

$$
\begin{array}{r}
e_{h}^{1}+\delta t C_{h}^{T} \varepsilon_{h}^{1}=i_{h} \tilde{e}_{h}^{0}+\delta t i_{h} R_{h}^{1}-\delta t i_{h} A_{h} \delta w_{h}^{1}-\delta t i_{h} A_{h} \tilde{e}_{h}^{0} \\
+\delta t C_{h}^{T} \delta q_{h}^{1}-\delta t i_{h} B_{h}^{T} \delta q_{h}^{1}-\delta t i_{h} B_{h}^{T} \epsilon_{h}^{0}, \\
\tilde{e}_{h}^{1}+\delta t A_{h} \tilde{e}_{h}^{1}=i_{h}^{T} e_{h}^{1}+\delta t A_{h} \tilde{e}_{h}^{0}-\delta t B_{h}^{T} B_{h} \tilde{e}_{h}^{0}+\delta t A_{h} \delta w_{h}^{1} .
\end{array}
$$

Taking the square for (4.33) and (4.34), we have

$$
\begin{aligned}
\left\|e_{h}^{1}\right\|_{0}^{2}+\delta t^{2}\left\|C_{h}^{T} \varepsilon_{h}^{1}\right\|_{0}^{2} & \lesssim\left\|\tilde{e}_{h}^{0}\right\|_{0}^{2}+\delta t^{2}\left\|R_{h}^{1}\right\|_{0}^{2}+\delta t^{2}\left\|A_{h} \delta w_{h}^{1}\right\|_{0}^{2}+\delta t^{2}\left\|A_{h} \tilde{e}_{h}^{0}\right\|_{0}^{2} \\
& +\delta t^{2}\left\|C_{h}^{T} \delta q_{h}^{1}\right\|_{0}^{2}+\delta t^{2}\left\|B_{h}^{T} \delta q_{h}^{1}\right\|_{0}^{2}+\delta t^{2}\left\|B_{h}^{T} \epsilon_{h}^{0}\right\|_{0}^{2},
\end{aligned}
$$

and

$$
\begin{aligned}
\left\|\tilde{e}_{h}^{1}\right\|_{0}^{2}+\delta t^{2}\left\|A_{h} \tilde{e}_{h}^{1}\right\|_{0}^{2}+\delta t\left\|\tilde{e}_{h}^{1}\right\|_{1}^{2} & \lesssim\left\|e_{h}^{1}\right\|_{0}^{2}+\delta t^{2}\left\|A_{h} \delta w_{h}^{1}\right\|_{0}^{2} \\
& +\delta t^{2}\left\|A_{h} \tilde{e}_{h}^{0}\right\|_{0}^{2}+\delta t^{2}\left\|B_{h}^{T} B_{h} \tilde{e}_{h}^{0}\right\|_{0}^{2} .
\end{aligned}
$$

From the initialization hypothesis ( $(\mathrm{H1})$, we obtain

$$
\left\|\tilde{e}_{h}^{1}\right\|_{0}^{2}+\left\|\tilde{e}_{h}^{0}\right\|_{0}^{2}+\delta t\left\|\tilde{e}_{h}^{1}\right\|_{1}^{2}+\delta t^{2}\left\|C_{h}^{T} \varepsilon_{h}^{1}\right\|_{0}^{2}+\delta t^{2}\left\|A_{h} \tilde{e}_{h}^{1}\right\|_{0}^{2} \lesssim \delta t^{2}\left(\delta t+h^{l+1}\right)^{2} .
$$

Collecting the above results yields the desired bound

$$
\begin{aligned}
\left\|\delta \tilde{e}_{h}^{n+1}\right\|_{0}^{2} & +\delta t^{2}\left\|D_{h}^{t} \tilde{e}_{h}^{n+1}\right\|_{0}^{2}+\delta t\left\|B_{h} \tilde{e}_{h}^{n+1}\right\|_{0}^{2} \\
& +\sum_{k=1}^{n}\left(\left\|i_{h}^{T} \delta e_{h}^{k+1}-\delta \tilde{e}_{h}^{k+1}\right\|_{0}^{2}+\left\|\delta^{2} \tilde{e}_{h}^{k+1}\right\|_{0}^{2}\right) \leq \delta t^{2}\left(\delta t+h^{l+1}\right)^{2} .
\end{aligned}
$$


Finally, the bound on $\tilde{e}_{h}-i_{h}^{T} e_{h}$ can be obtained from (4.6) as follows:

$$
\begin{aligned}
\left\|\tilde{e}_{h}^{n+1}-i_{h}^{T} e_{h}^{n+1}\right\|_{0} & =\delta t\left\|D_{h}^{t} \tilde{e}_{h}^{n+1}-A_{h} \delta w_{h}^{n+1}\right\|_{0} \\
& \lesssim \delta t\left\|D_{h}^{t} \tilde{e}_{h}^{n+1}\right\|_{0}+\delta t^{2} \lesssim \delta t\left(\delta t+h^{l+1}\right) .
\end{aligned}
$$

This completes the proof.

Remark 4.3. The estimate (4.25) is remarkable in the sense that, even though the time stepping scheme is only first-order, the discrete divergence of $\tilde{u}_{h}$ is $\frac{3}{2}$ order with respect to time. Actually, the $\frac{3}{2}$-order holds also if we replace the first-order backward Euler time stepping with the second-order BDF2 (i.e., $R_{h}^{k+1} \sim$ $\left.O\left(\delta t^{2}+h^{l+1}\right)\right)$. That the splitting error can be smaller than the consistency error induced by the time stepping has also been observed in [9]. This $\frac{3}{2}$-order on the discrete divergence of $\tilde{u}_{h}$ is the key reason why the second-order rotational scheme yields better error estimate for velocity in the $H^{1}$-norm and the pressure in the $L^{2}$-norm than the standard version of the algorithm (compare Theorem 3.1 and Theorem 5.1).

We are now in position to prove the major result of this section:

Theorem 4.1. Let $u_{h}, \tilde{u}_{h}, p_{h}$ solve (4.5)-(4.6) (or the equivalent algorithm (4.9)-

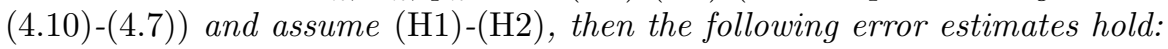

$$
\begin{aligned}
& \left\|u-\tilde{u}_{h}\right\|_{\ell^{2}\left(L^{2}(\Omega)^{d}\right)}+\left\|u-i_{h}^{T} u_{h}\right\|_{\ell^{2}\left(L^{2}(\Omega)^{d}\right)} \lesssim \delta t+h^{l+1}, \\
& \left\|u-\tilde{u}_{h}\right\|_{\ell^{2}\left(H^{1}(\Omega)^{d}\right)}+\left\|u-i_{h}^{T} u_{h}\right\|_{\ell^{2}\left(H^{1}(\Omega)^{d}\right)}+\left\|p-p_{h}\right\|_{\ell^{2}\left(L^{2}(\Omega)\right)} \lesssim \delta t+h^{l} .
\end{aligned}
$$

Proof. We reconstruct a non-homogeneous Stokes equation for the errors $\tilde{e}, \epsilon$, by subtracting (4.7) from (4.17),

$$
\left\{\begin{array}{l}
A_{h} \tilde{e}_{h}^{k+1}+B_{h}^{T} \epsilon_{h}^{k+1}=R_{h}^{k+1}-\frac{\tilde{e}_{h}^{k+1}-\tilde{e}_{h}^{k}}{\delta t}, \\
B_{h} \tilde{e}_{h}^{k+1}=-B_{h} \tilde{u}_{h}^{k+1} .
\end{array}\right.
$$

Standard stability results on non-homogeneous Stokes problems yield

$$
\left\|\tilde{e}_{h}^{k+1}\right\|_{1}+\left\|\epsilon_{h}^{k+1}\right\|_{0} \leq\left\|R_{h}^{k+1}\right\|_{-1}+\frac{1}{\delta t}\left\|\tilde{e}_{h}^{k+1}-\tilde{e}_{h}^{k}\right\|_{-1}+\left\|B_{h} \tilde{u}_{h}^{k+1}\right\|_{0} .
$$

Owing to Lemma 4.2, we have

$$
\begin{aligned}
\frac{1}{\delta t^{2}}\left\|\tilde{e}_{h}^{k+1}-\tilde{e}_{h}^{k}\right\|_{\ell^{2}\left(H^{-1}(\Omega)^{d}\right)}^{2} & \lesssim \frac{1}{\delta t^{2}}\left\|\tilde{e}_{h}^{k+1}-\tilde{e}_{h}^{k}\right\|_{\ell^{2}\left(L^{2}(\Omega)^{d}\right)}^{2} \lesssim \delta t^{2}+h^{2 l+2}, \\
\left\|B_{h} \tilde{u}_{h}^{k+1}\right\|_{\ell^{2}\left(L^{2}(\Omega)^{d}\right)} & \leq\left\|B_{h} \tilde{e}_{h}^{k+1}\right\|_{\ell^{\infty}\left(L^{2}(\Omega)^{d}\right)} \lesssim \delta t^{3 / 2}+\delta t^{1 / 2} h^{l+1} .
\end{aligned}
$$

This immediately implies

$$
\left\|\tilde{e}_{h}\right\|_{\ell^{2}\left(H^{1}(\Omega)^{d}\right)}+\left\|\epsilon_{h}\right\|_{\ell^{2}\left(L^{2}(\Omega)\right)} \lesssim \delta t+h^{l+1} .
$$

Moreover, using the Poincaré inequality we infer $\|\tilde{e}\|_{l^{2}\left(l^{2}(\Omega)^{d}\right)} \lesssim \delta t+h^{l+1}$, which in turn together with (4.23) yield $\left\|i_{h}^{T} e\right\|_{l^{2}\left(l^{2}(\Omega)^{d}\right)} \lesssim \delta t+h^{l+1}$. The desired results are then consequences of Lemma 4.1 .

Remark 4.4. The $\ell^{2}$ discrete norm in time in the estimates (4.35)-(4.36) can be replaced by the $\ell^{\infty}$-norm with a little more regularity asumption on the solution. We refer, e.g., to [10, Theorem 4.1] where such estimates are proven for the fully discrete standard form of the pressure-correction method. 


\section{SECOND-ORDER Rotational VELOCITY-CORRECTION SCHEME}

We now focus our attention on the fully discrete rotational velocity-correction algorithm with BDF2 in time. To shorten the presentation we only give the proof of the stability of the algorithm and we just mention the final convergence result. The technical details are similar to those in the proof of Theorem 4.1 plus a duality argument involving the right inverse of the Stokes operator (details can be found in 9,24$)$.

Replacing the Euler time stepping in (4.5) and (4.7) by BDF2 yields the following algorithm: Find $\left(\tilde{u}_{h}^{k+1}, p_{h}^{k+1}, u_{h}^{k+1}\right) \in\left(X_{h}, M_{h}, Y_{h}\right)$ such that

$$
\left\{\begin{array}{l}
\frac{3 u_{h}^{k+1}-4 i_{h} \tilde{u}_{h}^{k}+i_{h} \tilde{u}_{h}^{k-1}}{2 \delta t}+i_{h} A_{h} \tilde{u}_{h}^{k}-C_{h}^{T} B_{h} \tilde{u}_{h}^{k}+C_{h}^{T} p_{h}^{k+1} \\
+\left(i_{h} B_{h}^{T}-C_{h}^{T}\right) p_{h}^{k}=i_{h} f_{h}^{k+1}, \\
C_{h} u_{h}^{k+1}=0,
\end{array}\right.
$$

and

$$
\frac{3 \tilde{u}_{h}^{k+1}-4 \tilde{u}_{h}^{k}+\tilde{u}_{h}^{k-1}}{2 \delta t}+A_{h} \tilde{u}_{h}^{k+1}+B_{h}^{T} p_{h}^{k+1}=f_{h}^{k+1} .
$$

Lemma 5.1. The solution of the scheme (5.1)-(5.2) is bounded in the following sense:

$$
\left\|\delta \tilde{u}_{h}\right\|_{l^{\infty}\left(L^{2}(\Omega)^{d}\right)}+\delta t^{\frac{1}{2}}\left\|B_{h} \tilde{u}_{h}\right\|_{l^{\infty}\left(L^{2}(\Omega)\right)} \leq\left\|\delta f_{h}\right\|_{l^{2}\left(L^{2}(\Omega)^{d}\right)} .
$$

Proof. For simplicity we omit the source term $f$ since it does not affect the stability of the algorithm. We proceed as in the proof of Lemma 4.1.

Applying $i_{h}^{T}$ to (5.1) and subtracting the result from (5.2), we obtain

$$
\frac{3\left(\tilde{u}_{h}^{k+1}-i_{h}^{T} u_{h}^{k+1}\right)}{2 \delta t}+A_{h} \tilde{u}_{h}^{k+1}-A_{h} \tilde{u}_{h}^{k}+B_{h}^{T} B_{h} \tilde{u}_{h}^{k}=0 .
$$

After applying the time increment operator $\delta$ to (5.1), (5.2) and (5.4), we have

$$
\begin{gathered}
\left\{\begin{array}{c}
3 \delta u_{h}^{k+1}+2 \delta t C_{h}^{T} \phi_{h}^{k+1}=i_{h}\left(4 \delta \tilde{u}_{h}^{k}-\delta \tilde{u}_{h}^{k-1}\right) \\
-2 \delta t i_{h} D_{h}^{t} \tilde{u}_{h}^{k}+2 \delta t\left(C_{h}^{T}-i_{h} B_{h}^{T}\right) \phi_{h}^{k}, \\
C_{h} \delta u_{h}^{k+1}=0,
\end{array}\right. \\
3 \delta \tilde{u}_{h}^{k+1}-4 \delta \tilde{u}_{h}^{k}+\delta \tilde{u}_{h}^{k-1}+2 \delta t D_{h}^{t} \tilde{u}_{h}^{k+1}=-2 \delta t B_{h}^{T} \phi_{h}^{k+1}, \\
3 \delta \tilde{u}_{h}^{k+1}+2 \delta t D_{h}^{t} \delta \tilde{u}_{h}^{k+1}=3 i_{h}^{T} \delta u_{h}^{k+1} .
\end{gathered}
$$

The entire stability analysis is based on the equations (5.5)-(5.6)-(5.7) above. In the following, we will square each of them, sum up the results, use the two inequalities

$$
\left\|B_{h} v_{h}\right\|_{0} \leq\left\|\nabla v_{h}\right\|_{0}, \quad \forall v_{h} \in X_{h} ; \quad\left\|i_{h}^{T} y_{h}\right\|_{0} \leq\left\|y_{h}\right\|_{0}, \forall y_{h} \in Y_{h},
$$

and apply the discrete Gronwall lemma to the resulted inequality.

First, we square (5.5) to obtain

$$
\begin{gathered}
9\left\|\delta u_{h}^{k+1}\right\|_{0}^{2}+4 \delta t^{2}\left\|C_{h}^{T} \phi_{h}^{k+1}\right\|_{0}^{2}=\left\|4 \delta \tilde{u}_{h}^{k}-\delta \tilde{u}_{h}^{k-1}\right\|_{0}^{2}+4 \delta t^{2}\left\|D_{h}^{t} \tilde{u}_{h}^{k}\right\|_{0}^{2} \\
-4 \delta t\left(4 \delta \tilde{u}_{h}^{k}-\delta \tilde{u}_{h}^{k-1}, D_{h}^{t} \tilde{u}_{h}^{k}\right)+4 \delta t^{2}\left\|\left(C_{h}^{T}-i_{h} B_{h}^{T}\right) \delta \phi_{h}^{k}\right\|_{0}^{2} .
\end{gathered}
$$


Note that we used the fact that $\left(i_{h} v_{h},\left(C_{h}^{T}-i_{h} B_{h}^{T}\right) q_{h}\right)=0$ for all $v_{h} \in X_{h}$ and all $q_{h} \in M_{h}$, since $i_{h}^{T} i_{h}$ is the identity and $i_{h}^{T} C_{h}^{T}=B_{h}^{T}$. Then, we square (5.6) to get

$$
\begin{aligned}
& \left\|3 \delta \tilde{u}_{h}^{k+1}-4 \delta \tilde{u}_{h}^{k}+\delta \tilde{u}_{h}^{k-1}\right\|_{0}^{2}+4 \delta t^{2}\left\|D_{h}^{t} \tilde{u}_{h}^{k+1}\right\|_{0}^{2} \\
& \quad+4 \delta t\left(3 \delta \tilde{u}_{h}^{k+1}-4 \delta \tilde{u}_{h}^{k}+\delta \tilde{u}_{h}^{k-1}, D_{h}^{t} \tilde{u}_{h}^{k+1}\right)=4 \delta t^{2}\left\|B_{h}^{T} \phi_{h}^{k+1}\right\|_{0}^{2} .
\end{aligned}
$$

Finally, squaring (5.7) leads to

$$
9\left\|\delta \tilde{u}_{h}^{k+1}\right\|_{0}^{2}+4 \delta t^{2}\left\|D_{h}^{t} \delta \tilde{u}_{h}^{k+1}\right\|_{0}^{2}+4 \delta t\left(3 \delta \tilde{u}_{h}^{k+1}, D_{h}^{t} \delta \tilde{u}_{h}^{k+1}\right)=9\left\|i_{h}^{T} \delta u_{h}^{k+1}\right\|_{0}^{2} .
$$

Now we sum up (5.9)-(5.10)-(5.11) to obtain

$$
\begin{aligned}
9\left\|\delta u_{h}^{k+1}\right\|_{0}^{2} & +4 \delta t^{2}\left(\left\|C_{h}^{T} \phi_{h}^{k+1}\right\|_{0}^{2}-\left\|B_{h}^{T} \phi_{h}^{k+1}\right\|_{0}^{2}\right) \\
& +4 \delta t^{2}\left\|D_{h}^{t} \tilde{u}_{h}^{k+1}\right\|_{0}^{2}+9\left\|\delta \tilde{u}_{h}^{k+1}\right\|_{0}^{2}+I_{1}+I_{2} \\
& +4 \delta t^{2}\left\|D_{h}^{t} \delta \tilde{u}_{h}^{k+1}\right\|_{0}^{2}=4 \delta t^{2}\left\|D_{h}^{t} \tilde{u}_{h}^{k}\right\|_{0}^{2} \\
& +4 \delta t^{2}\left\|\left(C_{h}^{T}-i_{h} B_{h}^{T}\right) \delta \phi_{h}^{k}\right\|_{0}^{2}+9\left\|i_{h}^{T} \delta u_{h}^{k+1}\right\|_{0}^{2},
\end{aligned}
$$

where, to simplify notation, we have set

$$
I_{1}:=\left\|3 \delta \tilde{u}_{h}^{k+1}-4 \delta \tilde{u}_{h}^{k}+\delta \tilde{u}_{h}^{k-1}\right\|_{0}^{2}-\left\|4 \delta \tilde{u}_{h}^{k}-\delta \tilde{u}_{h}^{k-1}\right\|_{0}^{2},
$$

and

$$
\begin{aligned}
I_{2}:=4 \delta t\left[\left(3 \delta \tilde{u}_{h}^{k+1}, D_{h}^{t} \delta \tilde{u}_{h}^{k+1}\right)\right. & +\left(3 \delta \tilde{u}_{h}^{k+1}-4 \delta \tilde{u}_{h}^{k}+\delta \tilde{u}_{h}^{k-1}, D_{h}^{t} \tilde{u}_{h}^{k+1}\right) \\
& \left.+\left(4 \delta \tilde{u}_{h}^{k}-\delta \tilde{u}_{h}^{k-1}, D_{h}^{t} \tilde{u}_{h}^{k}\right)\right] .
\end{aligned}
$$

Observing that $\left\|C_{h}^{T} \phi_{h}^{k+1}\right\|_{0}^{2}-\left\|B_{h}^{T} \phi_{h}^{k+1}\right\|_{0}^{2}=\left\|\left(C_{h}^{T}-i_{h} B_{h}^{T}\right) \phi_{h}^{k+1}\right\|_{0}^{2}$ and $\left\|i_{h}^{T} \delta u_{h}^{k+1}\right\|_{0} \leq$ $\left\|\delta u_{h}^{k+1}\right\|_{0}$, (5.12) can be rewritten as

$$
\begin{array}{r}
9\left\|\delta \tilde{u}_{h}^{k+1}\right\|_{0}^{2}+I_{1}+I_{2}+4 \delta t^{2}\left\|\left(C_{h}^{T}-i_{h} B_{h}^{T}\right) \phi_{h}^{k+1}\right\|_{0}^{2}+4 \delta t^{2}\left\|D_{h}^{t} \tilde{u}_{h}^{k+1}\right\|_{0}^{2} \\
\quad+4 \delta t^{2}\left\|D_{h}^{t} \delta \tilde{u}_{h}^{k+1}\right\|_{0}^{2} \leq 4 \delta t^{2}\left\|D_{h}^{t} \tilde{u}_{h}^{k}\right\|_{0}^{2}+4 \delta t^{2}\left\|\left(C_{h}^{T}-i_{h} B_{h}^{T}\right) \delta \phi_{h}^{k}\right\|_{0}^{2} .
\end{array}
$$

Now we compute the terms $I_{1}$ and $I_{2}$. For $I_{1}$, we have

$$
\begin{aligned}
9\left\|\delta \tilde{u}_{h}^{k+1}\right\|_{0}^{2}+I_{1} & =3\left\|\delta \tilde{u}_{h}^{k+1}\right\|_{0}^{2}+3\left\|2 \delta \tilde{u}_{h}^{k+1}-\delta \tilde{u}_{h}^{k}\right\|_{0}^{2}+3\left\|\delta^{3} \tilde{u}_{h}^{k+1}\right\|_{0}^{2} \\
& -3\left\|\delta \tilde{u}_{h}^{k}\right\|_{0}^{2}-3\left\|2 \delta \tilde{u}_{h}^{k}-\delta \tilde{u}_{h}^{k-1}\right\|_{0}^{2} .
\end{aligned}
$$

The term $I_{2}$ can be simplified as follows:

$$
I_{2}=4 \delta t\left[\left(3 \delta \tilde{u}_{h}^{k+1}, D_{h}^{t} \tilde{u}_{h}^{k+1}\right)+\left(3 \delta \tilde{u}_{h}^{k+1}-4 \delta \tilde{u}_{h}^{k}+\delta \tilde{u}_{h}^{k-1}, D_{h}^{t} \delta \tilde{u}_{h}^{k+1}\right)\right] .
$$

Then, using the identity $2(3 a-4 b+c, a-b)=5(a-b)^{2}+(a-2 b+c)^{2}-(b-c)^{2}$ together with the definition of $D_{h}^{t}$, we infer

$$
\begin{aligned}
\frac{1}{\delta t} I_{2} & =12\left\|\nabla \delta \tilde{u}_{h}^{k+1}\right\|_{0}^{2}+12\left(\delta \tilde{u}_{h}^{k+1}, B_{h}^{T} B_{h} \tilde{u}_{h}^{k}\right) \\
& +4\left(3 \delta \tilde{u}_{h}^{k+1}-4 \delta \tilde{u}_{h}^{k}+\delta \tilde{u}_{h}^{k-1}, A_{h} \delta^{2} \tilde{u}_{h}^{k+1}+B_{h}^{T} B_{h} \delta \tilde{u}_{h}^{k}\right) \\
& =12\left\|\nabla \delta \tilde{u}_{h}^{k+1}\right\|_{0}^{2}+10\left\|\nabla \delta^{2} \tilde{u}_{h}^{k+1}\right\|_{0}^{2}+2\left\|\nabla \delta^{3} \tilde{u}_{h}^{k+1}\right\|_{0}^{2}-2\left\|\nabla \delta^{2} \tilde{u}_{h}^{k}\right\|_{0}^{2} \\
& +6\left\|B_{h} \tilde{u}_{h}^{k+1}\right\|_{0}^{2}-6\left\|B_{h} \tilde{u}_{h}^{k}\right\|_{0}^{2}-6\left\|B_{h} \delta^{2} \tilde{u}_{h}^{k+1}\right\|_{0}^{2} \\
& -8\left\|B_{h} \delta \tilde{u}_{h}^{k}\right\|_{0}^{2}-2\left\|B_{h} \delta^{2} \tilde{u}_{h}^{k}\right\|_{0}^{2}+2\left\|B_{h} \delta \tilde{u}_{h}^{k-1}\right\|_{0}^{2} .
\end{aligned}
$$


Thanks to the fact that $\left\|B_{h} v_{h}\right\|_{0} \leq\left\|\nabla v_{h}\right\|_{0}$ for all $v_{h} \in X_{h}$, we deduce

$$
\begin{aligned}
\frac{1}{\delta t} I_{2} & \geq 4\left\|\nabla \delta \tilde{u}_{h}^{k+1}\right\|_{0}^{2}+8\left(\left\|\nabla \delta \tilde{u}_{h}^{k+1}\right\|_{0}^{2}-\left\|\nabla \delta \tilde{u}_{h}^{k}\right\|_{0}^{2}\right) \\
& +4\left(\left\|\nabla \delta^{2} \tilde{u}_{h}^{k+1}\right\|_{0}^{2}-\left\|\nabla \delta^{2} \tilde{u}_{h}^{k}\right\|_{0}^{2}\right)+6\left(\left\|B_{h} \tilde{u}_{h}^{k+1}\right\|_{0}^{2}-\left\|B_{h} \tilde{u}_{h}^{k}\right\|_{0}^{2}\right) .
\end{aligned}
$$

Combining (5.13) with (5.14) and (5.15), we finally obtain

$$
\begin{aligned}
3\left\|\delta \tilde{u}_{h}^{k+1}\right\|_{0}^{2} & +6\left\|B_{h} \tilde{u}_{h}^{k+1}\right\|_{0}^{2}+3\left\|2 \delta \tilde{u}_{h}^{k+1}-\delta \tilde{u}_{h}^{k}\right\|_{0}^{2}+8\left\|\nabla \delta \tilde{u}_{h}^{k+1}\right\|_{0}^{2}+4\left\|\nabla \delta^{2} \tilde{u}_{h}^{k+1}\right\|_{0}^{2} \\
& +4 \delta t^{2}\left\|\left(C_{h}^{T}-i_{h} B_{h}^{T}\right) \phi_{h}^{k+1}\right\|_{0}^{2}+4 \delta t^{2}\left\|D_{h}^{t} \tilde{u}_{h}^{k+1}\right\|_{0}^{2} \\
& +3\left\|\delta^{2} \tilde{u}_{h}^{k+1}\right\|_{0}^{2}+4\left\|\nabla \delta \tilde{u}_{h}^{k+1}\right\|_{0}^{2}+4 \delta t^{2}\left\|D_{h}^{t} \delta \tilde{u}_{h}^{k+1}\right\|_{0}^{2} \\
& \left.\leq 3\left\|\delta \tilde{u}_{h}^{k}\right\|_{0}^{2}+6\left\|B_{h} \tilde{u}_{h}^{k}\right\|_{0}^{2}\right)+3\left\|2 \delta \tilde{u}_{h}^{k}-\delta \tilde{u}_{h}^{k-1}\right\|_{0}^{2}+8\left\|\nabla \delta \tilde{u}_{h}^{k}\right\|_{0}^{2}+4\left\|\nabla \delta^{2} \tilde{u}_{h}^{k}\right\|_{0}^{2} \\
& +4 \delta t^{2}\left\|\left(C_{h}^{T}-i_{h} B_{h}^{T}\right) \delta \phi_{h}^{k}\right\|_{0}^{2}+4 \delta t^{2}\left\|D_{h}^{t} \tilde{u}_{h}^{k}\right\|_{0}^{2} .
\end{aligned}
$$

The conclusion then follows readily by using the discrete Gronwall lemma.

Theorem 5.1. Under appropriate regularity assumptions and initialization hypotheses, the solution to (5.1)-(5.2) satisfies

$$
\begin{aligned}
& \left\|u-\tilde{u}_{h}\right\|_{\ell^{2}\left(L^{2}(\Omega)^{d}\right)}+\left\|u-i_{h}^{T} u_{h}\right\|_{\ell^{2}\left(L^{2}(\Omega)^{d}\right)} \lesssim \delta t^{2}+h^{l+1}, \\
& \left\|u-\tilde{u}_{h}\right\|_{\ell^{2}\left(H^{1}(\Omega)^{d}\right)}+\left\|p-p_{h}\right\|_{\ell^{2}\left(L^{2}(\Omega)\right)} \lesssim \delta t^{3 / 2}+h^{l} .
\end{aligned}
$$

Proof. Proceed as in the proof of Lemma 5.1 and use the right inverse of the discrete stokes operator as in [9, 12, 24].

Remark 5.1. Note that the estimate (5.17) is $\frac{1}{2}$-order suboptimal with respect to $\delta t$. This phenomenon is also observed for the rotational form of the pressure-correction method. It has been analyzed for the pressure-correction method in [12. The lack of optimality is related to the smoothness of the boundary. Actually, if the domain is a two-dimensional channel with one periodic direction, it has been shown in 2], using the normal mode analysis, that the rotational pressure-correction method is fully second-order. In the general case, if the boundary of the domain is smooth, say of class $\mathcal{C}^{1}$, numerical evidences reported in 12 show that the method is also fully second-order. But, if the boundary of the domain is only piecewise $\mathcal{C}^{1}$, say $\Omega$ is a convex rectangle, then the $\delta t^{\frac{1}{2}}$ suboptimality manifests itself in the convergence tests. This tends to confirm that our analysis is sharp under the assumption the domain is such that $H^{2}$ regularity holds for the steady Stokes problem supplemented with homogeneous Dirichlet boundary conditions and $L^{2}$ right-hand sides. Whether rotational pressure-correction and rotational velocity-correction methods can be modified to yield provable full second-order in any circumstance is still, to our best knowledge, an open problem; see [13] for additional details.

Remark 5.2. Here again we only derived the $\ell^{2}$-in-time estimates. It is possible to obtain $\ell^{\infty}$-in-time estimates by asssuming more regularity. We refer for instance to [9. Theorem 4.2] where this type of argument is developed for the standard version the pressure-correction method.

\section{Concluding Remarks}

The results in Theorems 4.1 and 5.1 show that the first-order and second-order rotational velocity-correction yield optimal error estimates in space for both the velocity and the pressure, provided that the inf-sup condition is satisfied. The time 
estimates are optimal for the velocity in the $L^{2}$-norm for both schemes. These estimates are also optimal in the $H^{1}$-norm for the first-order time stepping but are suboptimal by a $\delta t^{\frac{1}{2}}$ factor for the BDF2 time stepping. All these results are consistent with the numerical results presented in [12].

The present analysis holds for all types of approximations provided the assumptions (2.8) and (2.17)-(2.18) are satisfied. In particular, these conditions are satisfied by most finite element settings for spectral approximations though the story is slightly different. Although there are at least two pairs of spectral approximation spaces that satisfy the inf-sup condition (2.8) uniformly with respect to the polynomial degree $N$ (cf. [1]), the most popular pair $P_{N} \times P_{N-2}$ only satisfies a weaker inf-sup condition,

$$
\inf _{q_{h} \in M_{h}} \sup _{v_{h} \in X_{h}} \frac{\left(\nabla \cdot v_{h}, q_{h}\right)}{\left\|\nabla v_{h}\right\|_{0}} \geq c_{h}\left\|q_{h}\right\|_{0},
$$

where $c_{h}:=\beta_{N}=N^{\frac{1-d}{2}} \rightarrow 0$ as $N \rightarrow \infty(d=2$ or 3 is the dimension; see, for instance, [1]). Although this does not affect the derivation of $\delta t$-estimates, it does introduce difficulties for proving $\delta t^{2}$-estimates on the velocity for the second-order schemes, since the constant $c_{h}$ comes into play when we apply the right-inverse of the discrete Stokes operator, leading to an estimate of the form

$$
\left\|u-\tilde{u}_{h}\right\|_{\ell^{2}\left(L^{2}(\Omega)^{d}\right)} \lesssim c_{h}^{-1}\left(\delta t^{2}+h^{l+1}\right) .
$$

Numerical tests reported in [11, 12] indicate that the term $c_{h}^{-1}$ does not affects the accuracy on the velocity and should not be present in (6.2). How to remove the term $c_{h}^{-1}$ in the above error estimate for spectral approximations is still an open problem.

\section{REFERENCES}

[1] C. Bernardi and Y. Maday, Uniform inf-sup conditions for the spectral discretization of the Stokes problem, Math. Models Methods Appl. Sci. 9 (1999), no. 3, 395-414. MR 1686546 (2000b:65208)

[2] David L. Brown, Ricardo Cortez, and Michael L. Minion, Accurate projection methods for the incompressible Navier-Stokes equations, J. Comput. Phys. 168 (2001), no. 2, 464-499. MR.1826523 (2002a:76112)

[3] A. J. Chorin, Numerical solution of the Navier-Stokes equations, Math. Comp. 22 (1968), 745-762. MR0242392 (39:3723)

[4] W. E and J. G. Liu, Projection method I: Convergence and numerical boundary layers, SIAM J. Numer. Anal. 32 (1995), 1017-1057. MR1342281 (96e:65061)

[5] Weinan E and Jian-Guo Liu, Gauge method for viscous incompressible flows, Commun. Math. Sci. 1 (2003), no. 2, 317-332. MR.1980478 (2004c:76039)

[6] V. Girault and P. A. Raviart, Finite element methods for Navier-Stokes equations, SpringerVerlag, 1986. MR851383 (88b:65129)

[7] K. Goda, A multistep technique with implicit difference schemes for calculating two- or three-dimensional cavity flows, J. Comput. Phys. 30 (1979), 76-95.

[8] J. L. Guermond, Some implementations of projection methods for Navier-Stokes equations, RAIRO Modél. Math. Anal. Numér. 30 (1996), no. 5, 637-667. MR1411394 (97h:76086)

[9] J.-L. Guermond, Un résultat de convergence d'ordre deux en temps pour l'approximation des équations de Navier-Stokes par une technique de projection incrémentale, M2AN Math. Model. Numer. Anal. 33 (1999), no. 1, 169-189; also in C. R. Acad. Sci. Paris, Série I, 325:1329-1332, 1997. MR2000k:65171

[10] J.-L. Guermond and L. Quartapelle, On the approximation of the unsteady Navier-Stokes equations by finite element projection methods, Numer. Math. 80 (1998), no. 5, 207-238. MR1645029 (99i:65105) 
[11] J. L. Guermond and Jie Shen, A new class of truly consistent splitting schemes for incompressible flows, J. Comput. Phys. 192 (2003), no. 1, 262-276. MR2045709 (2005k:76076)

[12] _ Velocity-correction projection methods for incompressible flows, SIAM J. Numer. Anal. 41 (2003), no. 1, 112-134 (electronic). MR.1974494 (2004c:65103)

[13] J. L. Guermond, P. Minev, and Jie Shen, An overview of projection methods for incompressible flows, Comput. Methods Appl. Mech. Engrg. 195 (2006), 6011-6045. MR2250931 (2007g:76157)

[14] J. L. Guermond and Jie Shen, On the error estimates of rotational pressure-correction projection methods, Math. Comp 73 (2004), 1719-1737. MR2059733 (2005f:65113)

[15] J. G. Heywood and R. Rannacher, Finite element approximation of the nonstationary NavierStokes problem. I. Regularity of solutions and second-order error estimates for spatial discretization, SIAM J. Numer. Anal. 19 (1982), 275-311. MR650052 (83d:65260)

[16] _ Finite element approximation of the nonstationary Navier-Stokes problem. III. Smoothing property and higher order estimates for spatial discretization, SIAM J. Numer. Anal. 25 (1988), 489-512. MR942204 (89k:65114)

[17] Hans Johnston and Jian-Guo Liu, Accurate, stable and efficient Navier-Stokes solvers based on explicit treatment of the pressure term, J. Comput. Phys. 199 (2004), no. 1, 221-259. MR:2081004 (2005b:76093)

[18] G. E. Karniadakis, M. Israeli, and S. A. Orszag, High-order splitting methods for the incompressible Navier-Stokes equations, J. Comput. Phys. 97 (1991), 414-443. MR1137607 (92h:76066)

[19] G. E. Karniadakis and S. J. Sherwin, Spectral/hp element methods for cfd, Oxford University Press, 1999. MR 1696933 (2000h:76120)

[20] J. Kim and P. Moin, Application of a fractional-step method to incompressible Navier-Stokes equations, J. Comput. Phys. 59 (1985), 308-323. MR796611 (87a:76046)

[21] Ricardo H. Nochetto and Jae-Hong Pyo, Error estimates for semi-discrete gauge methods for the Navier-Stokes equations, Math. Comp. 74 (2005), no. 250, 521-542 (electronic). MR2114636 (2005i:65132)

[22] S. A. Orszag, M. Israeli, and M. Deville, Boundary conditions for incompressible flows, J. Sci. Comput. 1 (1986), 75-111.

[23] Andreas Prohl, Projection and quasi-compressibility methods for solving the incompressible Navier-Stokes equations, Advances in Numerical Mathematics, B.G. Teubner, Stuttgart, 1997. MR1472237 (98k:65058)

[24] J. Shen, On error estimates of projection methods for the Navier-Stokes equations: Secondorder schemes, Math. Comp. 65 (1996), no. 215, 1039-1065. MR.1348047(96j:65091)

[25] J. Shen and Xiaofeng Yang, Error estimates for finite element approximations of consistent splitting schemes for incompressible flows, DCDS-B 8 (2007), 663-676. MR.2328729

[26] Jie Shen, On error estimates of the projection methods for the Navier-Stokes equations: First-order schemes, SIAM J. Numer. Anal. 29 (1992), 57-77. MR1149084 (92m:35213)

[27] R. Temam, Sur l'approximation de la solution des équations de Navier-Stokes par la méthode des pas fractionnaires II, Arch. Rat. Mech. Anal. 33 (1969), 377-385. MR0244654 (39:5968)

[28] L. J. P. Timmermans, P. D. Minev, and F. N. Van De Vosse, An approximate projection scheme for incompressible flow using spectral elements, Int. J. Numer. Methods Fluids 22 (1996), 673-688.

[29] J. van Kan, A second-order accurate pressure-correction scheme for viscous incompressible flow, SIAM J. Sci. Stat. Comput. 7 (1986), 870-891. MR848569 (87h:76008)

Department of Mathematics, Texas A\&M University, College Station, Texas 77843

E-mail address: guermond@math.tamu.edu

Department of Mathematics, Purdue University, West Lafayette, Indiana 47907

E-mail address: shen@math.purdue.edu

Department of Mathematics, University of North Carolina, Chapel Hill, NC 27599

E-mail address: xfyang@unc.edu 